\title{
OPEN Human infrapatellar fat pad mesenchymal stem cells show immunomodulatory exosomal signatures
}

\author{
Dimitrios Kouroupis ${ }^{1,2,3 凶}$, Lee D. Kaplan ${ }^{1} \&$ Thomas M. Best $^{1}$
}

Within the human knee infrapatellar fat pad (IFP) and synovium, resident synoviocytes and macrophages contribute to the onset and progression of inflammatory joint diseases. Our hypothesis is that IFP-derived mesenchymal stem cells (IFP-MSC) robust immunomodulatory therapeutic effects are largely exerted via their exosomal (IFP-MSC EXOs) secretome by attenuating synoviocytes and macrophages pro-inflammatory activation. IFP-MSC EXOs showed distinct miRNA and protein immunomodulatory profiles. Reactome analysis of 24 miRNAs highly present in exosomes showed their involvement in the regulation of six gene groups, including immune system. Exosomes were enriched for immunomodulatory and reparative proteins that are involved in positive regulation of cell proliferation, response to stimulus, signal transduction, signal receptor activity, and protein phosphorylation. Stimulated synoviocytes or macrophages exposed to IFP-MSC EXOs demonstrated significantly reduced proliferation, altered inflammation-related molecular profiles, and reduced secretion of pro-inflammatory molecules compared to stimulated alone. In an acute synovial/ IFP inflammation rat model, IFP-MSC EXOs therapeutic treatment resulted in robust macrophage polarization towards an anti-inflammatory therapeutic M2 phenotype within the synovium/IFP tissues. Based on these findings, we propose a viable cell-free alternative to MSC-based therapeutics as an alternative approach to treating synovitis and IFP fibrosis.

The synovium and IFP combine as a single anatomic and functional unit ${ }^{1}$ and serve as a site of immune cell infiltration and origin of pro-inflammatory and articular cartilage (AC) degradative molecules. In pathological conditions, deranged chondrocyte metabolism results in release within the joint of AC degradation products and other damage-associated molecular patterns (DAMPs) ${ }^{2-5}$ that incite local responses within the synovium/IFP tissues. These responses include: (1) local infiltration of immune cells such as macrophages, (2) shift of resident macrophages to a pro-inflammatory M1 phenotype ${ }^{2,6}$, (3) production of AC-degrading proteases (e.g., MMPs), and pro-inflammatory cytokines, adipokines and other mediators, and (4) sustained synovitis and progressive IFP fibrosis.

Mesenchymal Stem Cell (MSC)-based therapy has gained attention as a potential therapeutic alternative for such inflammatory conditions, given their immunomodulatory and trophic effects involving anti-inflammatory and anti-fibrotic actions ${ }^{7}$. IFP tissue constitutes a promising alternative source of MSC to other adult/foetal tissues such as the "standard" bone marrow (BM), given its anatomical relationship with intra-articular structures and its pivotal role in joint homeostasis and disease ${ }^{8}$. Based on this, we recently reported human IFP derived Mesenchymal Stem Cells (IFP-MSC) acquiring a potent immunomodulatory phenotype, and actively reversing inflammation and fibrosis linked with macrophage polarization from an M1 in disease to an M2 phenotype after engraftment into areas of active synovitis/IFP fibrosis ${ }^{9-14}$.

Exosomes are nanosized (50-200 nm) extracellular vesicles (EVs) generated via the endosomal pathway ${ }^{15}$, and secreted by numerous cells in response to their surrounding milieu. Thus, their contents (i.e., cargo) and lipid shell may carry information that reflects particular changes in the parental cells' microenvironment specifying intrinsic communications to proximal or distal sites. Previous studies have isolated and characterized exosomes

\footnotetext{
${ }^{1}$ Department of Orthopedics, UHealth Sports Medicine Institute, University of Miami, Miller School of Medicine, Miami, FL, USA. ${ }^{2}$ Diabetes Research Institute \& Cell Transplantation Center, University of Miami, Miller School of Medicine, Miami, FL, USA. ${ }^{3}$ Department of Orthopaedics, Division of Sports Medicine, Diabetes Research Institute, Cell Transplant Center, University of Miami, Miller School of Medicine, 1450NW 10th Ave, Room 3014, Miami, FL 33136, USA. ${ }^{\square}$ email: dxk504@med.miami.edu
} 
from various MSC sources (i.e. bone marrow, umbilical cord, adipose tissues), confirming their strong antiinflammatory, anti-fibrotic and angiogenesis-remodeling capacities ${ }^{16}$. Herein, our hypothesis is that IFP-MSC robust immunomodulatory effects are largely exerted via their exosomal secretome by attenuating synoviocytes and macrophages proliferation and pro-inflammatory activation. Our overarching goal is to determine whether the resulting IFP-MSC derived exosomes (IFP-MSC EXOs) are able to recapitulate our reported therapeutic activity with IFP-MSC ${ }^{9-13}$. Cell-free products have advantages compared with their replicative cell-based counterparts, including reduced variability, standardization, easy storage, superior safety profile and arguably a smoother regulatory landscape $e^{17,18}$, constituting a promising therapeutic alternative.

In the present study, we investigated IFP-MSC EXOs protein and miRNA signatures, and their effects on stimulated with inflammatory and fibrotic cues, synoviocytes, and macrophages. IFP-MSC show potent molecular and protein immunomodulatory exosomal profiles whereas IFP-MSC EXOs significantly affect synoviocytes and macrophages functionality in inflammatory conditions in vitro. Importantly, in an acute synovial/IFP inflammation rat model, therapeutic treatment with IFP-MSC EXOs promote robust macrophage polarization toward an anti-inflammatory therapeutic M2 phenotype within the synovium/IFP. These types of observations could provide a rationale for further testing of the IFP-MSC EXOs as a viable therapeutic modality to manufacture cell-free products for both synovitis/IFP fibrosis as well as chronic conditions such as osteoarthritis (OA) where inflammation is increasingly recognized as an important component of the disease pathology.

\section{Results and discussion}

IFP-MSC EXOs isolation and characterization. Our previous studies clearly demonstrated that IFPMSC manufacturing under regulatory-compliant conditions (human platelet lysate-hPL) results in a finetuned, regulatory-compliant, cell-based product with superior immunomodulatory properties that overcome any donor-to-donor variability in biological functionality ${ }^{19-21}$. On this basis, hPL-expanded IFP-MSC can efficiently degrade substance $P$ and suppress $T$ cell proliferation in vitro, whereas they can dramatically reverse signs of synovitis and IFP fibrosis upon intra-articular infusion in vivo ${ }^{20,21}$. Therefore, herein using our established IFP-MSC manufacturing protocol we investigated IFP-MSC EXOs protein and miRNA signatures, and their immunomodulatory/anti-inflammatory functionality in vitro.

As expected, IFP-MSC cultured in Ch-R medium showed fibroblast-like morphology (Fig. 1A) and high expression (>95\%) of common MSC-defining markers as established by ISCT ${ }^{22,23}$, including CD44, CD73, CD90, CD105, and CD107a (Fig. 1B). Similar to previous reports ${ }^{24}$, the CD146 and NG2 markers which define the pericytic origin of $\mathrm{MSC}^{25,26}$, were highly expressed (77.0 $\pm 3.0 \%$ and $92.5 \pm 2.7 \%$, respectively). Importantly, human leukocyte antigen HLA-DR was absent in all IFP-MSC donors. These data indicate that IFP-MSC retain their perivascular MSC-related immunophenotype without signs of immunogenicity in vitro.

IFP-MSC EXOs were characterized by size and surface marker expression following the guidelines recommended by ISEV (International Society for Extracellular Vesicles), termed MISEV2018 (Minimal Information for Studies of Extracellular Vesicles 2018) ${ }^{27}$ (Fig. 1C). Nanoparticle tracking analysis showed that isolated vesicles from IFP-MSC conditioned medium are $<200 \mathrm{~nm}$ diameter size, consistent with the known size of exosomes. IFP-MSC EXOs yield was $3-4 \times 10^{9} / \mathrm{ml}$ as detected by nanoparticle tracking analysis of all IFP-MSC donors. Also, $\mathrm{CD}^{+} 3^{+}$-selected exosomes showed purity by high positivity for exosomal marker CD9 (90.0 $\left.\pm 10.3 \%\right)$ (Fig. 1D).

miRNA and protein signature of IFP-MSC EXOs. MicroRNAs (miRNAs) are single-stranded RNA molecules that can influence cell functionality by binding to the $3^{\prime}$-UTR of cognate mRNAs, leading to the degradation or translational inhibition of target $\mathrm{mRNAs}^{28,29}$. Studies have demonstrated their immunomodulatory roles and their potential for inflammatory disease treatment ${ }^{30}$. In parallel, exosomes with specific miRNA cargos can be isolated from various joint tissues including infrapatellar fat pad that can influence whole joint homeostasis $^{31}$. Based on this, one study demonstrated that IFP-MSC EXOs highly abundant in miR-100-5p can effectively ameliorate gait abnormalities in OA mice and alleviate articular cartilage lesions in vivo ${ }^{32}$.

Herein, we investigated the MSC-related miRNA cargo of IFP-MSC EXOs cultured in Ch-R medium. From 166 MSC-related miRNAs assessed, 154 were present as cargo within IFP-MSC EXOs with 24 miRNAs being highly present (Fig. 2A). Furthermore, the first 4 out of 24 highly present miRNAs (hsa-miR-142-3p, hsa-miR146a, hsa-miR-107, hsa-miR-25-3p) show strong involvement in immune cell regulation. Interestingly, studies have shown that hsa-miR-142-3p attenuates phagocytosis and cytokine secretion of inflammatory mediators, including TNF- $\alpha$, IL-6, and IL-12p40, in myeloid inflammatory cells whereas it relieves neuropathic pain by targeting high mobility group box $1^{33,34}$. The second highly present miRNA cargo, hsa-miR-146a, belongs to the hsa-miR-146 family of genes that are expressed in response to pro-inflammatory stimuli as negative feedback to control excessive inflammation. Specifically, studies showed that hsa-miR-146a negatively regulates the adaptive immunity by modulating adaptor protein (AP)-1 activity and IL-2 expression in T cells, as well as immune cell activation and cytokine production ${ }^{35,36}$. In addition, the expression levels of hsa-miR-107 have been demonstrated to be related to TLR4 activation whereas hsa-miR-107 decreased expression may be a regulative feedback effect to limit insulin resistance in inflammation ${ }^{37}$. Studies have shown that has-miR-25-3p inhibits macrophage secretion of inflammatory cytokines in sepsis by targeting high mobility group box 1 whereas MSC EXOs cargo alleviates myocardial infarction by targeting pro-apoptotic proteins in vivo ${ }^{38,39}$. In the present study, reactome analysis of 24 miRNAs highly present in exosomes showed their involvement in the regulation of six gene groups related to the: immune system, NGF/PDGF/Wnt pathways, cell cycle, gene expression, cellular responses to stress, and homeostasis (Fig. 2B and Table 1). Overall, from a clinical standpoint the potent functionality of these highly present miRNAs can strongly support the notion of developing novel cell-free therapeutics for inflammation/ fibrosis reversal based on MSC secretome. Furthermore, miRNA interactome analysis revealed 6 miRNAs (hsamiR-98-5p, hsa-let-7e-5p, hsa-miR-21-5p, hsa-miR-15a-5p, hsa-miR-25-3p, hsa-miR-107) with higher node 
A

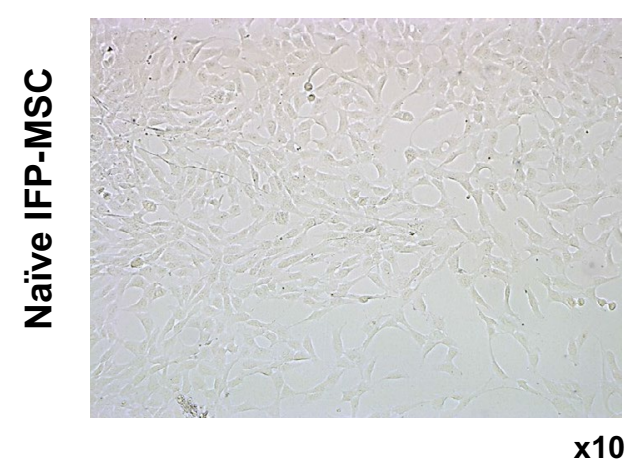

B

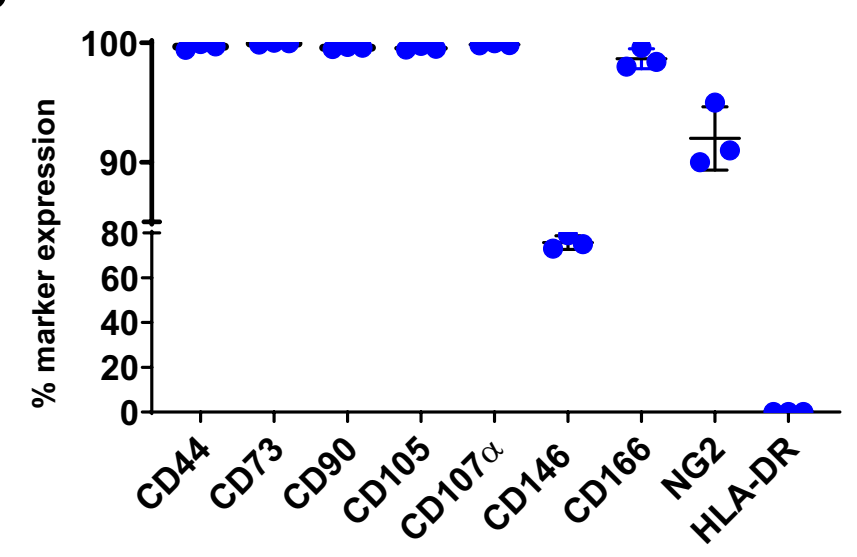

D

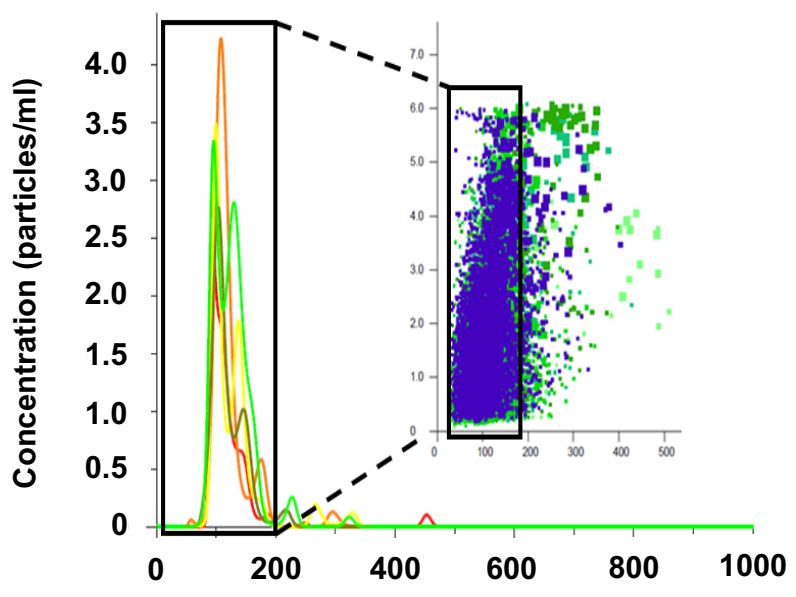

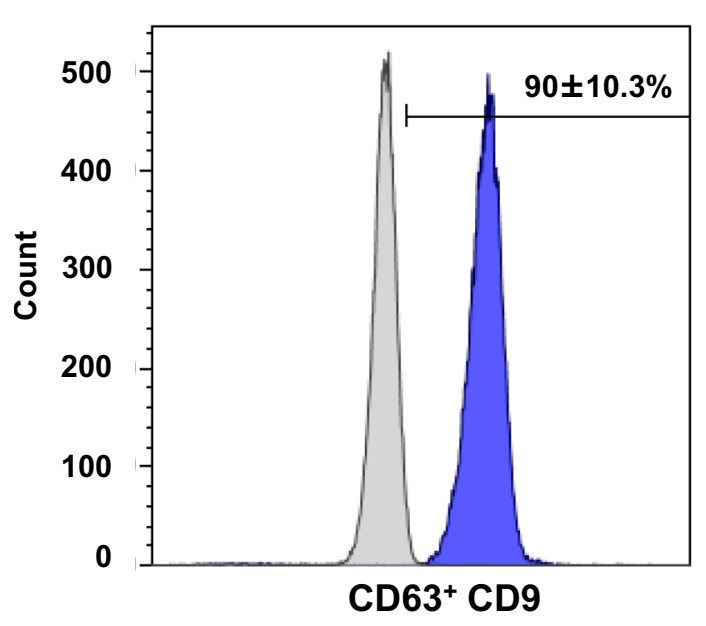

Figure 1. IFP-MSC culture and IFP-MSC EXOs isolation. (A, B) IFP-MSC showed a fibroblast-like morphology and high expression (>95\%) of common MSC-defining markers. Interestingly, IFP-MSC had high expression of CD146 (77.0 $\pm 3.0 \%)$ and NG2 $(92.5 \pm 2.7 \%)$ pericytic markers, whereas showed absence of human leukocyte antigen HLA-DR $(n=3)$. (C) Nanoparticle tracking analysis showed that isolated vesicles from IFP-MSC conditioned medium are $<200 \mathrm{~nm}$ diameter size, consistent with the known size of exosomes. (D) $\mathrm{CD}^{+}{ }^{+}$-selected exosomes showed purity by high positivity for exosomal marker CD9.

degree that act as hubs in a gene network (Supplementary Fig. 1). Even though the levels of these miRNAs as cargo within the IFP-MSC EXOs are variable, they control multiple genes related to important pathways such as Jak-STAT signaling pathway, focal adhesion, neurotrophin signaling pathway, p53 signaling pathway, cytokinecytokine receptor interaction and others.

We detected multiple immunomodulatory and reparative molecules secreted as a cargo within the IFPMSC EXOs (Fig. 3A). The exosomal "signature" involves the upregulated secretion of key immunomodulatory molecules including IL-6, IL-8, IP-10, TIMP-2. IL-6 and IL-8 cytokines have been implicated in both pro- and anti-inflammatory actions with previous studies indicating that IL-6/IL-8 ratio plays a crucial role in the specific polarization of the cellular microenvironment ${ }^{40}$. Importantly, studies have illustrated that IP-10 secretion directly correlates with decreased T cell proliferation ${ }^{41}$ whereas TIMP-2 attenuates the development of inflammation and inflammatory pain via MMP-dependent and receptor-mediated cell signaling mechanisms ${ }^{42}$. Specifically, NF- $\kappa B$, Nrf2, and CREB pathways are largely involved in TIMP-2-mediated anti-inflammatory effects ${ }^{43}$. In parallel, key reparative molecules including TGF- $\beta 2$, VEGFD, $\beta F G F$, IGF-I sR showed upregulated secretion in IFP-MSC EXOs. TGF- $\beta 2$ is a key molecule affecting cell proliferation, differentiation and apoptosis via various signal transduction networks such as serine/threonine receptors and SMAD protein phosphorylation. Of note, studies showed that TGF- $\beta 2$ can suppress IL-2-dependent T cell proliferation ${ }^{44}$. Interestingly, VEGF secreted by MSC induces endothelial progenitor cell differentiation towards endothelial cells via paracrine actions ${ }^{45}$.

All proteins appeared interconnected at least through one association and into three groups (red, green, blue, Fig. 3). All K-means clustering networks demonstrated elevated protein-protein interaction enrichment $(p<1.0 e-16)$ and an average local clustering coefficient $>0.7$ indicating that the proteins used are at least partially biologically connected (Fig. 3B). In terms of biological processes, various categories were highly affected 
A

154 IFP-MSC EXOs miRNAs

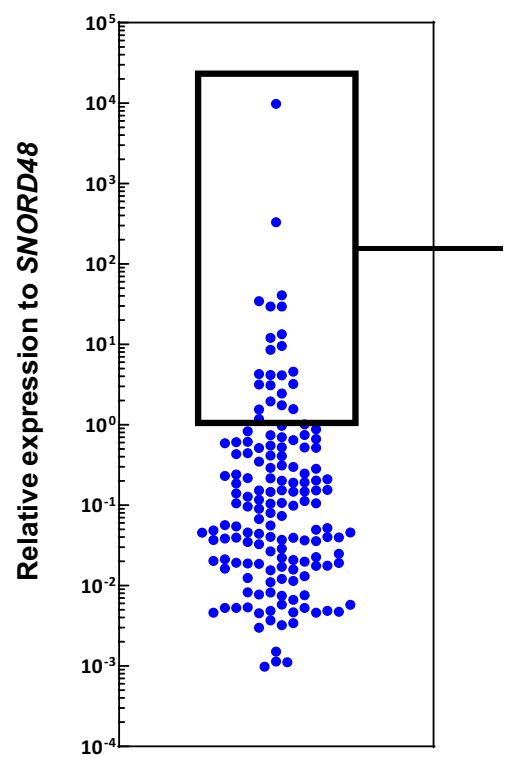

24 miRNAs highly present in IFP-MSC EXOs

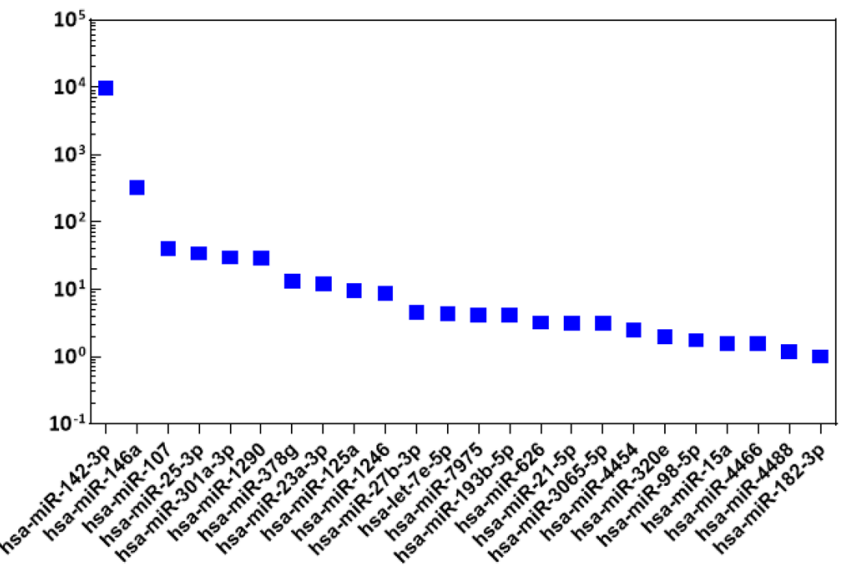

B

\section{Immune system (innate $\&$ adaptive)}

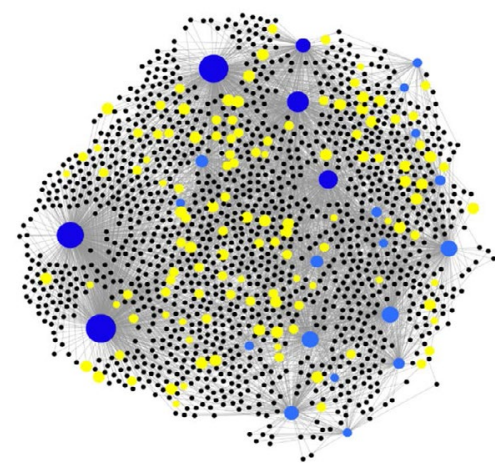

Gene expression

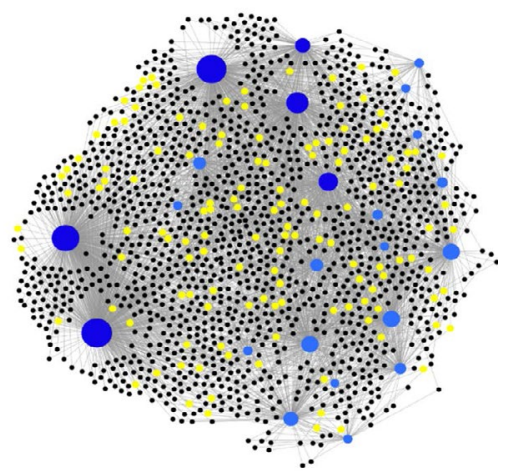

NGF, PDGF, Wnt pathways

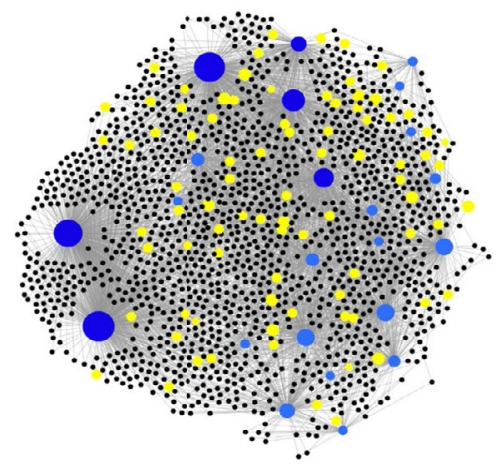

Cellular responses to stress

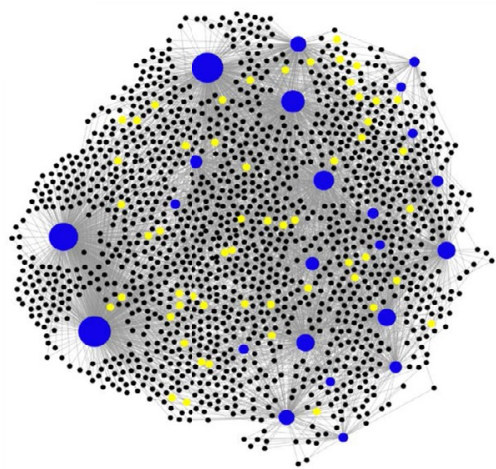

Cell cycle

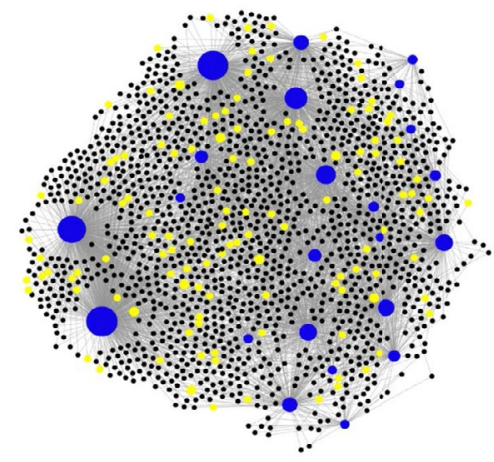

Homeostasis

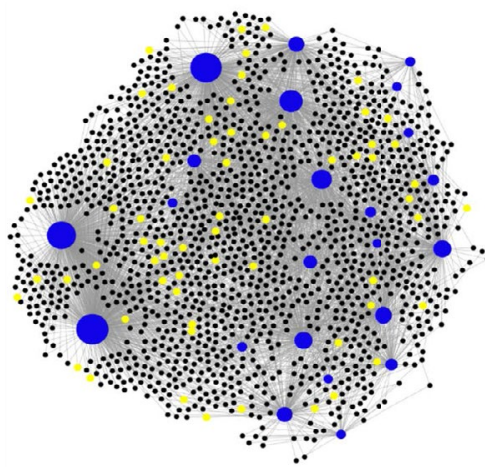

Figure 2. The miRNA signature of IFP-MSC EXOs. (A) 154 miRNAs were detected as cargo within IFP-MSC EXOs with 24 miRNAs being highly present. (B) Reactome analysis of 24 miRNAs highly present in exosomes showed their involvement in the regulation of six gene groups related to the: immune system, NGF/PDGF/Wnt pathways, cell cycle, gene expression, cellular responses to stress, and homeostasis. 


\begin{tabular}{|l|l|l|}
\hline Top reactome hits & Number of genes & Pval \\
\hline Gene expression & 134 & $1.12 \mathrm{E}-09$ \\
\hline Immune system & 123 & 0.00015 \\
\hline Innate immune system & 79 & 0.000428 \\
\hline Cell cycle & 67 & 0.00268 \\
\hline Hemostasis & 63 & 0.00135 \\
\hline Adaptive immune system & 62 & 0.000691 \\
\hline Cellular responses to stress & 55 & $6.18 \mathrm{E}-09$ \\
\hline Signaling by NGF & 52 & $9.64 \mathrm{E}-07$ \\
\hline NGF signaling via TRKA from the plasma membrane & 41 & $4.33 \mathrm{E}-07$ \\
\hline Generic transcription pathway & 41 & $4.33 \mathrm{E}-07$ \\
\hline Signaling by PDGF & 40 & $1.90 \mathrm{E}-07$ \\
\hline Signaling by Wnt & 40 & 0.000578 \\
\hline
\end{tabular}

Table 1. Reactome analysis of 24 miRNAs highly present in IFP-MSC EXOs.

including regulation of signal transduction, positive regulation of response to stimulus, regulation of signaling receptor activity, positive regulation of protein phosphorylation, positive regulation of cell population proliferation, positive regulation of cell migration, regulation of immune process, and angiogenesis. Combined, these effects empower IFP-MSC EXOs to respond to inflammation/injury by altering key cascades known to affect local immune responses. Regarding the type of signaling pathways affected, IFP-MSC EXOs have profound effects on cytokine-cytokine receptor interaction, signaling by interleukins, MAPK signaling, PI3K-Akt signaling pathway, Ras signaling, Rap1 signaling, Jak-STAT signaling, and interleukin-10 signaling (Fig. 3C). Both cytokine-cytokine receptor interaction and signaling by interleukins which are among the highest affected pathways play crucial role in regulating inflammatory and pain cascades. Similarly to IFP-MSC EXOs protein cargo, our previous published data showed that IFP-MSC soluble protein secretomes have significant involvement in cytokine-cytokine receptor interaction and signaling by interleukins ${ }^{20}$.

Therefore, both miRNA and protein cargo of IFP-MSC EXOs strongly indicate their potent functionality which upon further studies could result in the development of specialized cell-free therapies that overcome regulatory constrains for safe and effective regulation of inflammation and fibrosis in humans.

IFP-MSC EXOs effects on synoviocytes and macrophages. The histological profile of the synovium in OA preclinical models ${ }^{13}$ and in OA patients ${ }^{46}$ is mainly characterized by synovial lining hyperplasia and IFP fibrosis. The main cellular types contributing to these histological changes are synoviocytes and macrophages. Specifically, synoviocytes can be divided into type A (macrophage-like synoviocytes) which constitute resident macrophages, and type B (fibroblast-like synoviocytes) which display typical fibroblast markers such as surface marker Thy-1 (CD90) and are mainly responsible for maintenance of synovial homeostasis ${ }^{47,48}$. We separately investigated the effects of two IFP-MSC EXOs concentrations (EXO and $10 \times \mathrm{EXO}$ ) on naïve and stimulated synoviocytes in vitro in an effort to identify the optimal therapeutic dosage for in vivo application.

In co-cultures, IFP-MSC EXOs were internalized in both naïve and TIC-primed SYN whereas SYN and TIC SYN proliferation was attenuated by both EXO and $10 \times \mathrm{EXO}$ (Fig. $4 \mathrm{~A}, \mathrm{~B}$ ). This is an important finding suggesting to us that IFP-MSC EXOs could attenuate synovial lining hyperplasia upon their in vivo intra-articular infusion. Similarly, a previous study showed that synoviocytes exposure to BM-MSC EXOs results in suppressed rheumatoid arthritis-synoviocyte activation, migration, and invasion in vitro ${ }^{49}$. At the molecular level the TICSYN inflammation-related profile was altered by IFP-MSC EXOs. Of note, increased expression of $I L-1-\alpha$ and $I L-6$ may be counter balanced by increased $I L-8$ expression levels (Fig. 4 C). IL- 8 has been recognized to have anti-inflammatory activity, which has been established in various inflammation models. These molecular data are coupled by SYN secretory protein profiling in SYN/ IFP-MSC EXOs co-cultures. Overall, the secretion of immunomodulatory molecules was reduced 3.7-fold for EXO and 3.3-fold for 10 $\times$ EXO. Specifically, IL-6, IL-8, IP-10, MCP-1, MCP-2, RANTES, IFN- $\gamma$, TNF- $\alpha$, TIMP-2 were significantly $(p<0.05)$ reduced upon exposure to IFP-MSC EXOs compared to SYN stimulated alone (Fig. 4D). These findings indicate the strong capacity of the IFP-MSC secretome to attenuate not only the proliferation but also the pro-inflammatory activation of type $\mathrm{A}$ and type $\mathrm{B}$ synoviocytes in vitro.

In patients with OA, both the IFP and the synovium are infiltrated by various immune cells including monocytes/macrophages. These infiltrates complement the resident cells with macrophage polarization towards the pro-inflammatory classical M1 phenotype (reviewed in ${ }^{48}$ ). Specifically, in patients with OA, macrophages are recruited within the synovium in increased numbers compared to non OA patients and form clusters of multinucleated giant cells. Of note, studies have demonstrated a significant association between multinucleated giant cell numbers and synovitis severity ${ }^{46}$. On this basis, macrophage phenotype manipulation has been proposed as a potential therapy given that initial data indicate polarization of macrophages towards an alternative antiinflammatory M2 phenotype can be induced. Herein, we investigated the effects of two IFP-MSC EXOs concentrations (EXO and $10 \times \mathrm{EXO}$ ) on naïve and stimulated macrophages in vitro.

Similar to synoviocytes, naïve and PMA/IO-stimulated THP-1 macrophages didn't only internalized IFPMSC EXOs, but also attenuated their proliferation in both EXO and $10 \times \mathrm{EXO}$ concentrations (Fig. 5A,B). At the 
A

$$
\begin{aligned}
& \text { IFP-MSC EXOs } \\
& \text { protein cargo }
\end{aligned}
$$

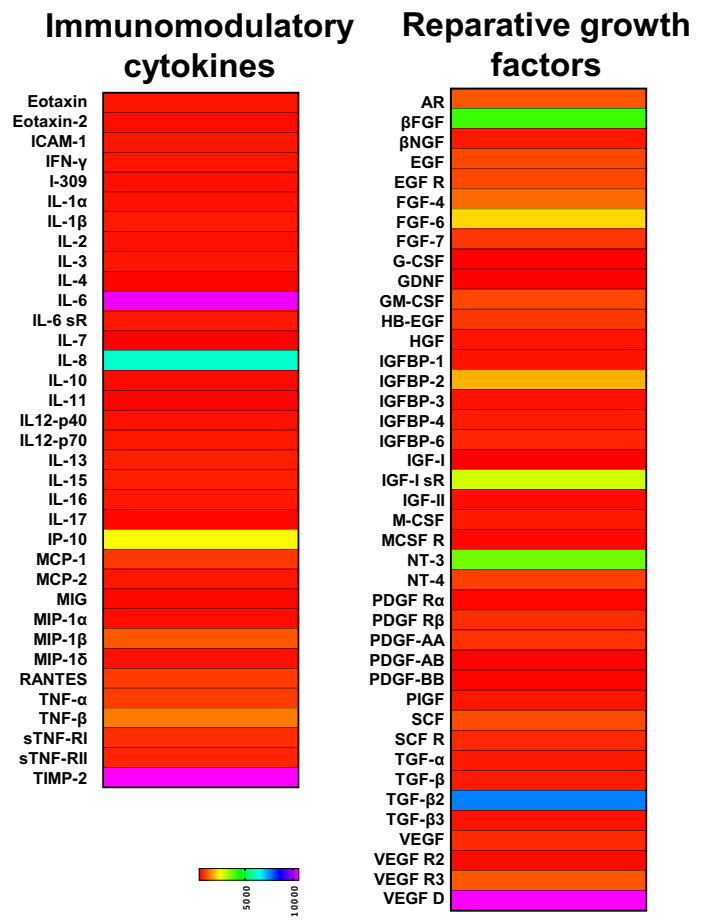

C

\section{Biological processes}

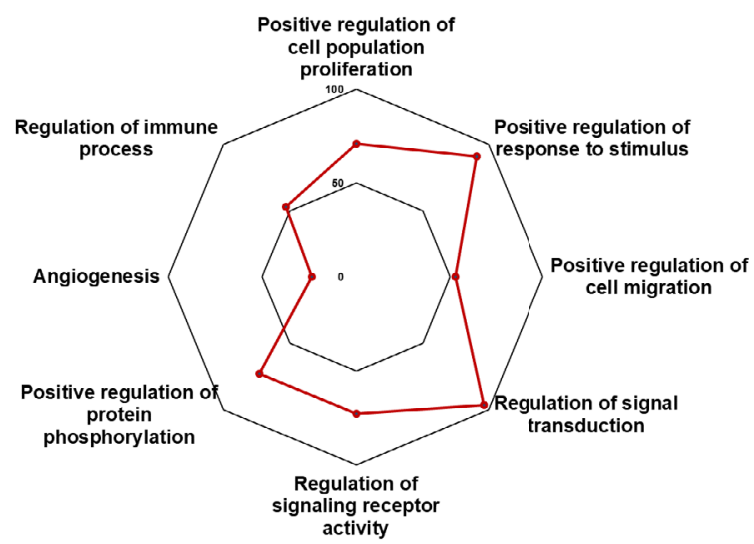

B

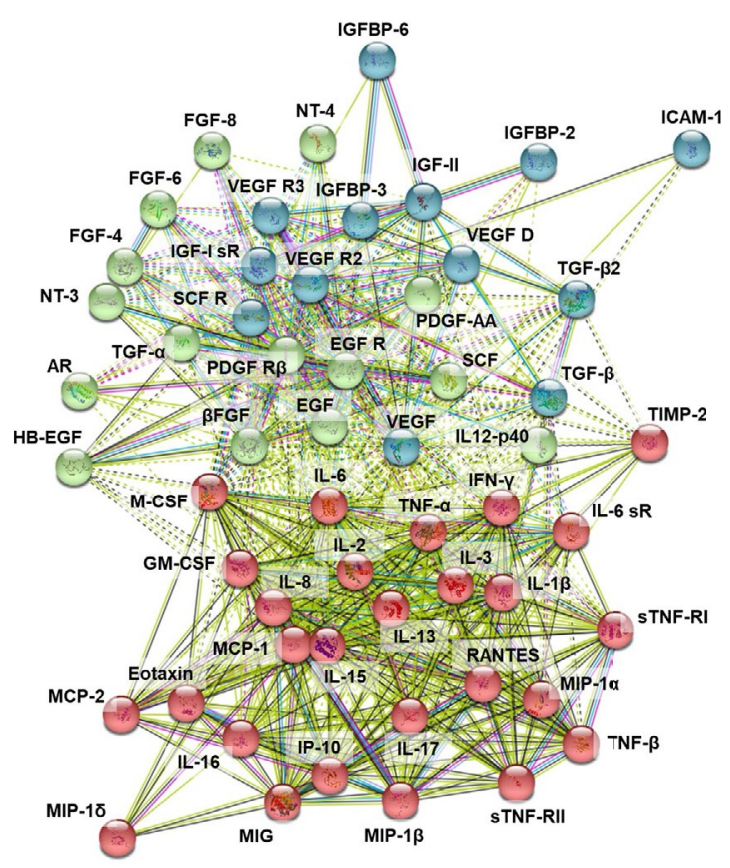

\section{Signaling pathways}

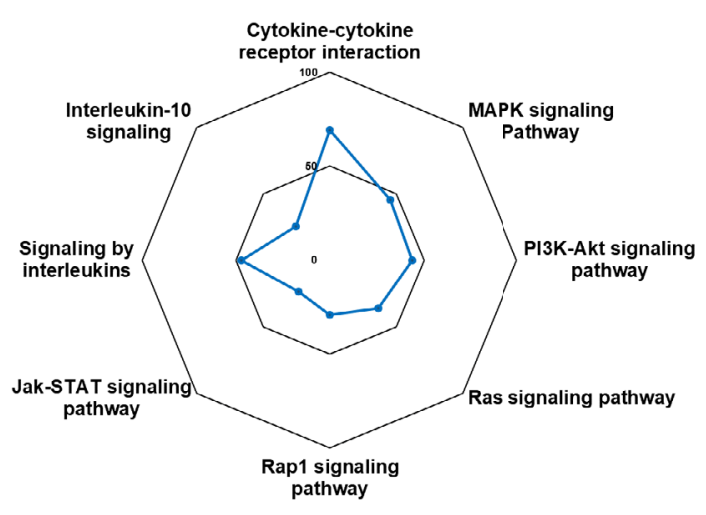

Figure 3. Protein signature of IFP-MSC EXOs. (A) IFP-MSC EXOs were enriched for immunomodulatory and reparative proteins. (B) All proteins appeared interconnected at least through one association and into three groups (red, green, blue). All K-means clustering networks demonstrated elevated protein-protein interaction (PPI) enrichment $(p<1.0 e-16)$ and an average local clustering coefficient $>0.7$ indicating that the proteins used are at least partially biologically connected. (C) Identified proteins are involved in multiple biological processes and pathway regulation in KEGG reactome analysis.

molecular level, PMA/IO-stimulated THP-1 molecular profiling indicated a strong gene expression shift towards an M2 macrophage polarization by both EXO and $10 \times \mathrm{EXO}$ (Fig. 5C). Most importantly, the expression levels of $M R C 1$ (Cd206) characteristic M2-polarization macrophages marker ${ }^{50}$ was strongly induced when macrophages were exposed to $10 \times \mathrm{EXO}$. CCL2 expression was also significantly induced in $10 \times \mathrm{EXO}$. CCL2 chemokine is associated with monocyte recruitment in inflamed tissues via CCR2 chemokine receptor after pro-inflammatory cytokines activation. Importantly, CCL2 and CCR 2 determine the extent of M2 macrophage polarization by enhancing the production of anti-inflammatory IL-10 cytokine ${ }^{51}$. Overall, the secretion of immunomodulatory 

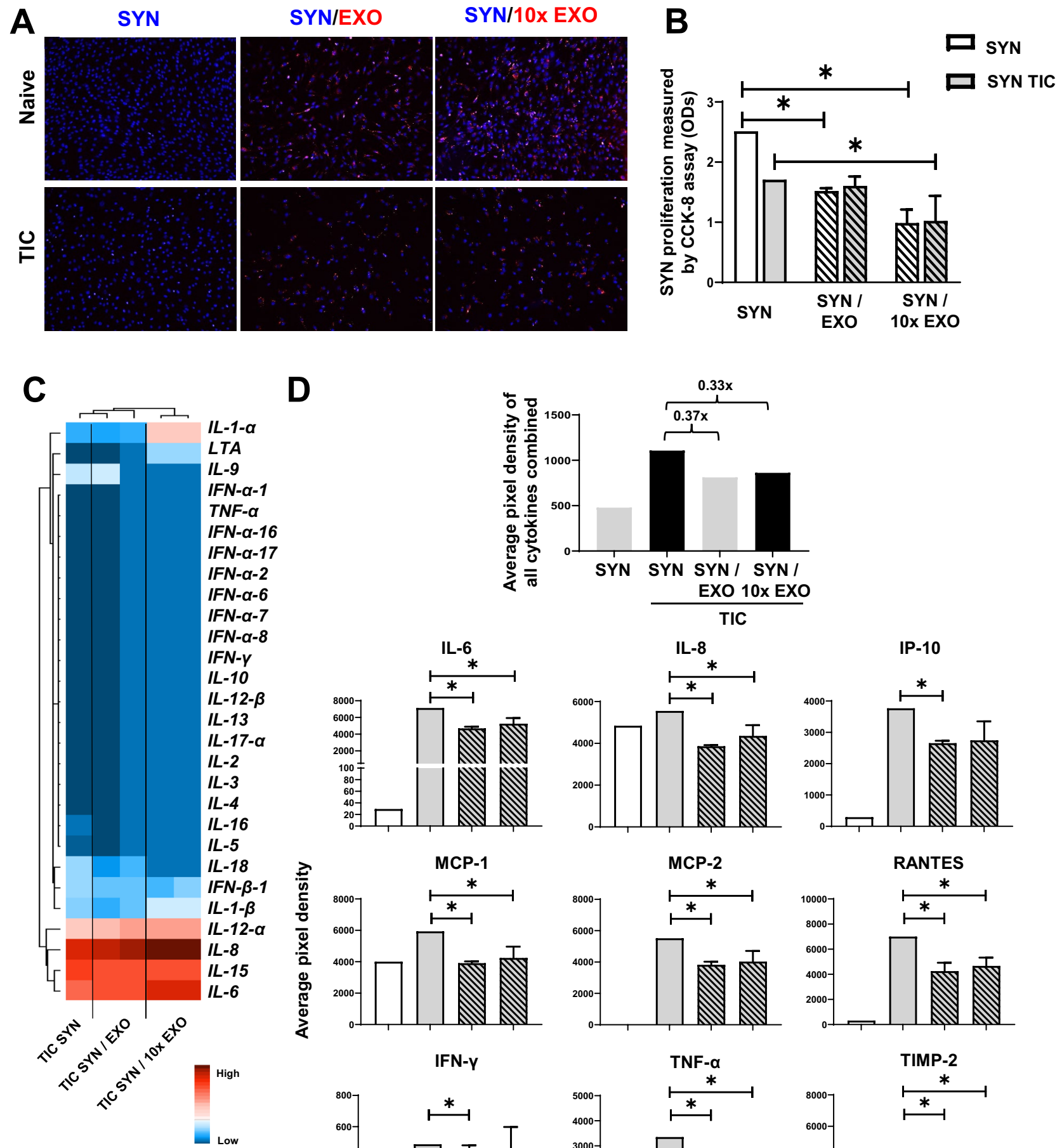

D
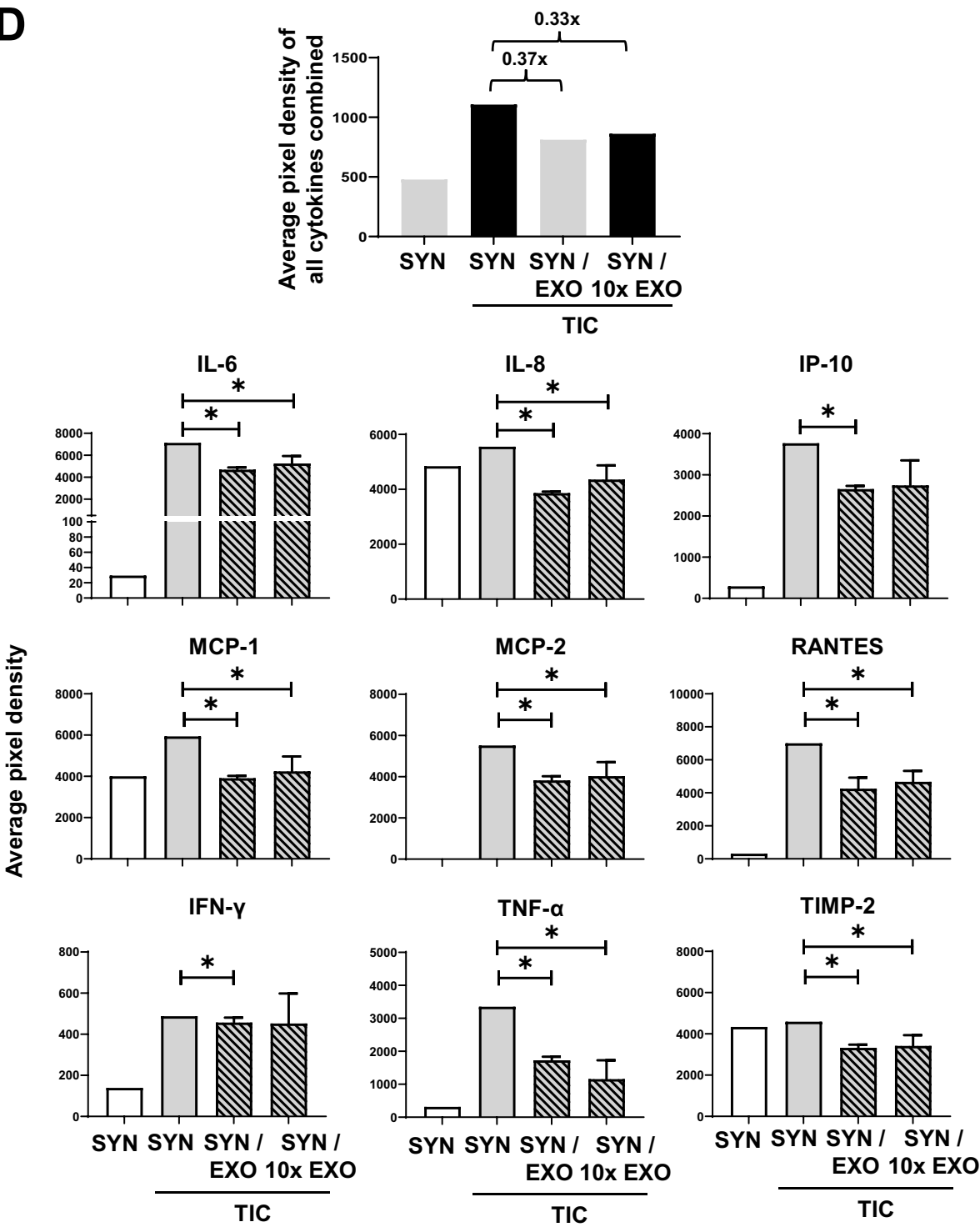

Figure 4. IFP-MSC EXOs anti-inflammatory effects on synoviocytes. (A) IFP-MSC EXOs were internalized in both naïve and TIC-primed SYN (blue, nucleus; red, EXOs). (B) SYN and TIC SYN proliferation was attenuated by both EXO and $10 \times$ EXO. (C) TIC SYN inflammation-related transcriptional profiling was altered by IFPMSC EXOs. (D) IFP-MSC EXOs significantly reduced secretion of pro-inflammatory molecules from TIC SYN compared to SYN stimulated alone $\left({ }^{*} p<0.05\right)$. 

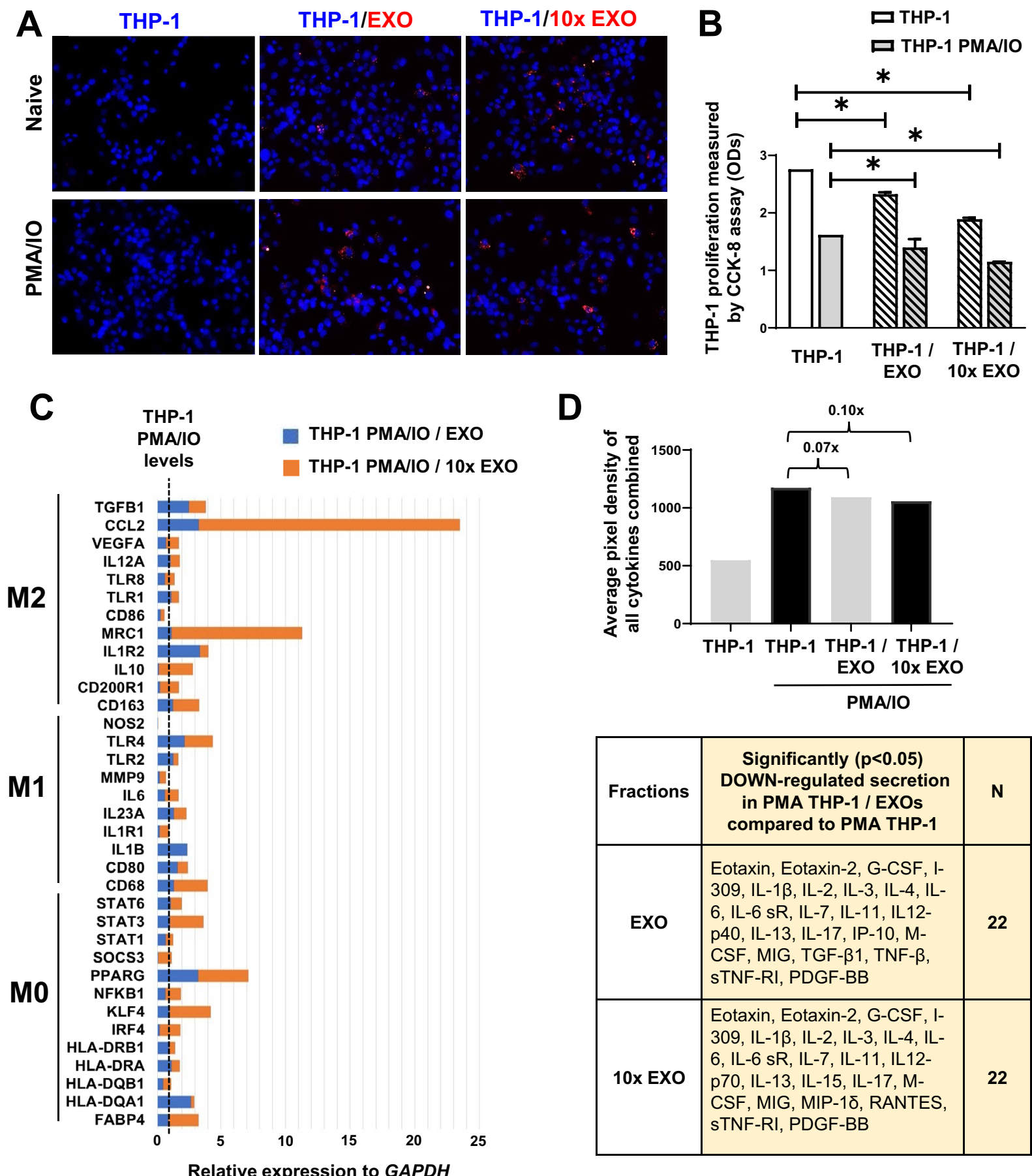

\begin{tabular}{|c|c|c|}
\hline Fractions & $\begin{array}{l}\text { Significantly }(p<0.05) \\
\text { DOWN-regulated secretion } \\
\text { in PMA THP-1 / EXOs } \\
\text { compared to PMA THP-1 }\end{array}$ & $\mathbf{N}$ \\
\hline EXO & $\begin{array}{l}\text { Eotaxin, Eotaxin-2, G-CSF, I- } \\
\text { 309, IL-1 } 1 \beta \text {, IL-2, IL-3, IL-4, IL- } \\
6 \text {, IL-6 sR, IL-7, IL-11, IL12- } \\
\text { p40, IL-13, IL-17, IP-10, M- } \\
\text { CSF, MIG, TGF- } \beta 1 \text {, TNF- } \beta \text {, } \\
\text { sTNF-RI, PDGF-BB }\end{array}$ & 22 \\
\hline 10x EXO & 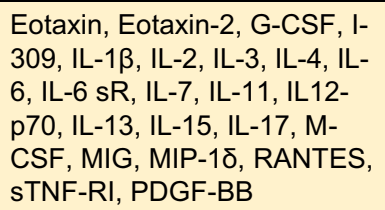 & 22 \\
\hline
\end{tabular}

Figure 5. IFP-MSC EXOs immunomodulatory effects on macrophages. (A) IFP-MSC EXOs were internalized in both naïve and PMA/IO-stimulated THP-1 macrophages (blue, nucleus; red, EXOs). (B) THP-1 and PMA/ IO-stimulated THP-1 proliferation was attenuated by both EXO and 10 $\times$ EXO. (C) PMA/IO-stimulated THP-1 molecular profiling indicated a strong gene expression shift towards an M2 macrophage polarization by both EXO and $10 \times$ EXO. (D) IFP-MSC EXOs significantly reduced secretion of pro-inflammatory molecules from PMA/IO-stimulated THP-1 compared to stimulated macrophages alone $\left({ }^{*} p<0.05\right)$.

molecules was reduced $0.07 \mathrm{x}$ for EXO and $0.1 \mathrm{x}$ for $10 \times$ EXO. Specifically, we observed a significant $(p<0.05)$ down-regulated secretion of 22 immunomodulatory proteins for both EXO and $10 \times$ EXO in PMA THP-1/EXO compared to PMA THP-1 (Fig. 5D). These findings indicate the significant effect of IFP-MSC EXOs on macrophages at both the molecular and protein levels. Collectively, these data are consistent with our previous reports indicating an M1 to M2 IFP macrophage phenotypic polarization following a single intra-articular injection of a subset of BM-MSC in rats with induced synovitis and IFP fibrosis ${ }^{52}$. 
IFP-MSC EXOs effects on synovitis, IFP fibrosis and macrophages polarization in vivo. An acute synovial/IFP inflammation rat (MIA) model was used to test the capacity of IFP-MSC EXOs upon intraarticular infusion to reverse synovial and IFP inflammation, IFP fibrosis, and macrophage polarization from M1 to M2 phenotypes (Fig. 6A).

Compared to healthy rat knees, the diseased group (only MIA) demonstrated strong synovitis $(157.0 \pm 26.5 \mu \mathrm{m}$ synovium thickness), characterized by synovial lining hyperplasia and IFP fibrosis ( $47.8 \pm 2.3 \%$ of fibrotic area) and characterized by loss of adipocytes within the main body of the IFP tissue (Fig. 6B,C). According to Udo et al. infrapatellar fat pad inflammation scoring (0-5) for rat arthritis in the MIA model ${ }^{53}$, only the diseased group developed grade 3 synovitis and grade 2 to 3 IFP fibrosis. Upon EXO or $10 \times$ EXO infusion, synovitis was significantly $(p<0.05)$ reduced $(95.0 \pm 23.9 \mu \mathrm{m}$ and $59.1 \pm 13.8 \mu \mathrm{m}$ synovium thickness, respectively) compared to the diseased group. Similarly, both EXO and $10 \times \mathrm{EXO}$ infusion resulted in increased $(p<0.05)$ fibrosis clearance from the IFP main body $(20.6 \pm 1.6 \%$ and $14.0 \pm 7.0 \%$ of fibrotic area, respectively). Interestingly, a previous study indicated that fibroblast-like synoviocytes contribute to fibrogenesis by excessive extracellular matrix deposition and enhanced contractile function ${ }^{54}$. Therefore, upon IFP-MSC EXOs infusion reduction of synovitis may be directly related to reduced IFP fibrosis in vivo.

Synovium/IFP resident and infiltrated macrophages have demonstrated key roles in joint homeostasis and articular cartilage degeneration, as they are the source of inflammatory and catabolic mediators such as IFN$\gamma$, TNF- $\alpha$, IL-1, IL-6, IL-8, and metalloproteinases ${ }^{48,55}$. On this basis, we performed classically activated-M1 $\left(\mathrm{CD}^{+}\right)$/alternatively activated-M2 (CD206 $\left.{ }^{+}\right)$macrophage phenotypic evaluations to determine mechanistic cellular changes promoted in synovium/IFP tissues after intra-articular IFP-MSC EXO infusion. Importantly, previous studies showed that parent MSCs can effectively modulate macrophage polarization in vivo ${ }^{52,56}$. In the present study, MIA infusion resulted in a significant $(p<0.05)$ shift of macrophage polarization towards the M1 phenotype (M1/M2 ratio: 9.6) from the homeostatic status of IFP tissue in healthy group (M1/M2 ratio: 1.1). Four days after IFP-MSC EXOs infusion, macrophage polarization was adversely shifted towards the alternatively activated-M2 phenotype (M1/M2 ratio: 0.3$)$, whereas the M1 phenotype was significantly $(p<0.05)$ reduced compared to the MIA group only (16,134 vs 46,179 AUs, respectively). Remarkably, $10 \times$ EXO infusion resulted in almost ten times stronger macrophage polarization towards the M2 phenotype (M1/M2 ratio: 0.07) compared to EXOs, with M1-polarized macrophage subpopulation reverting to homeostatic conditions $(2,212$ AUs in $10 \times \mathrm{EXO}$ and $1,790 \mathrm{AU}$ in healthy groups). These data are in accordance with a previous study indicating that a single intra-articular infusion of adipose tissue-derived MSC EXOs not only inhibits M1 macrophage infiltration into the synovium but also protects from articular cartilage degeneration ${ }^{57}$. Importantly, our present cell-free approach further reinforces our previous single-cell RNA-sequencing data which clearly demonstrated that macrophages within the IFP can shift towards a more immunomodulatory polarization status after a single IFP-MSC intra-articular therapy ${ }^{14}$. Therefore, both EXO and $10 \times \mathrm{EXO}$ treatments suggest robust macrophage polarization toward an anti-inflammatory therapeutic M2 phenotype within the synovium/IFP tissues.

\section{Conclusions}

In summary, IFP-MSC show potent molecular and protein immunomodulatory exosomal profiles that significantly affect synoviocytes and macrophages functionality in inflammatory conditions in vitro. Our results help elucidate various local activities of IFP-MSC EXOs within the IFP during joint disease pathogenesis and progression. However, this study has some limitations that deserve consideration. Specifically, the effects of IFP-MSC EXOs on T and B cell proliferation and maturation should be investigated in further experiments. Importantly, from a therapeutic perspective the local activities of intra-articularly infused EXOs may be related not only with the control of IFP fibrosis, but also with robust immune-related responses via shift of M1 to M2 macrophages polarization status. Therefore, based on our findings, we propose a proof-of-concept viable cell-free alternative to MSC-based therapeutics in the treatment of joint disease characterized by synovitis, IFP fibrosis, articular cartilage degradation, and potentially OA. Further studies are needed to strongly support the robust IFP-MSC EXOs therapeutic effects in various joint OA phenotypes.

\section{Materials and methods}

Isolation, culture and expansion of IFP-MSC. All experiments using human cells were performed in accordance with relevant guidelines and regulations. Human IFP-MSC were isolated from IFP tissue obtained from de-identified, non-arthritic patients that were age- and sex-matched $(n=5$; three males and two females; $36.6 \pm 12.9$ years old) undergoing elective knee arthroscopy at the Lennar Foundation Medical Center at the University of Miami. Informed consent was obtained from all participants and/or their legal guardians. All procedures were carried out following approval by the University of Miami IRB not as human research (based on the nature of the samples as discarded tissue). IFP tissue $(5-10 \mathrm{cc})$ was mechanically dissected and washed repeatedly with Dulbecco's Phosphate Buffered Saline (DPBS; Sigma Aldrich, St Louis, MO, USA), followed by enzymatic digestion using $235 \mathrm{U} / \mathrm{ml}$ Collagenase I (Worthington Industries, Columbus, OH, USA) diluted in DPBS and $1 \%$ bovine serum albumin (Sigma) for $2 \mathrm{~h}$ at $37^{\circ} \mathrm{C}$ with agitation. Enzymatic digestion was inactivated with complete media with DMEM low glucose GlutaMAX (ThermoFisher Scientific, Waltham, MA, USA) containing $10 \%$ fetal bovine serum (FBS; VWR, Radnor, PA, USA), washed and seeded at a density of $1 \times 10^{6}$ cells $/ 175 \mathrm{~cm}^{2}$ flask in chemically-reinforced (Ch-R) Mesenchymal Stem Cell Growth Medium 2 (PromoCell, Heidelberg, Germany). Forty-eight hours ( $48 \mathrm{~h}$ ) post-seeding, non-adherent cells were removed by DPBS gentle rinsing and fresh media were replenished accordingly.

All MSC were cultured at $37^{\circ} \mathrm{C} 5 \%(\mathrm{v} / \mathrm{v}) \mathrm{CO}_{2}$ until $80 \%$ confluent as passage $0(\mathrm{P} 0)$, then passaged at a 1:5 ratio until P3, detaching them with TrypLE ${ }^{\text {rx }}$ Select Enzyme 1X (Gibco, ThermoFisher Scientific) and assessing cell viability with $0.4 \%$ (w/v) Trypan Blue (Invitrogen, ThermoFisher Scientific). 
A

ACUTE SYNOVIAL/IFP INFLAMMATION MODEL

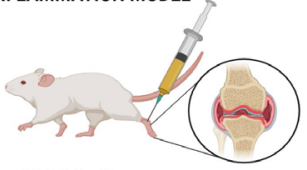

INTRA-ARTICULAR INJECTION OF EXOSOMES

MIA injection

$\checkmark$
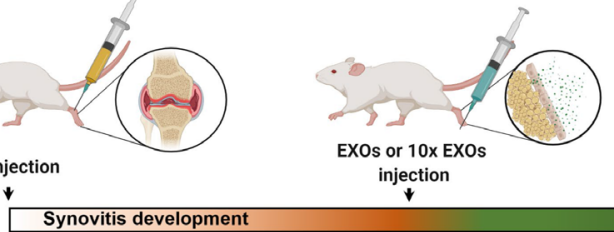

EXOs or 10x EXO injection

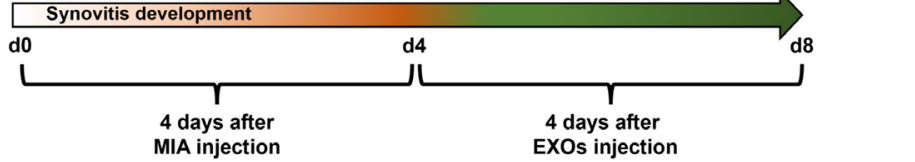

B
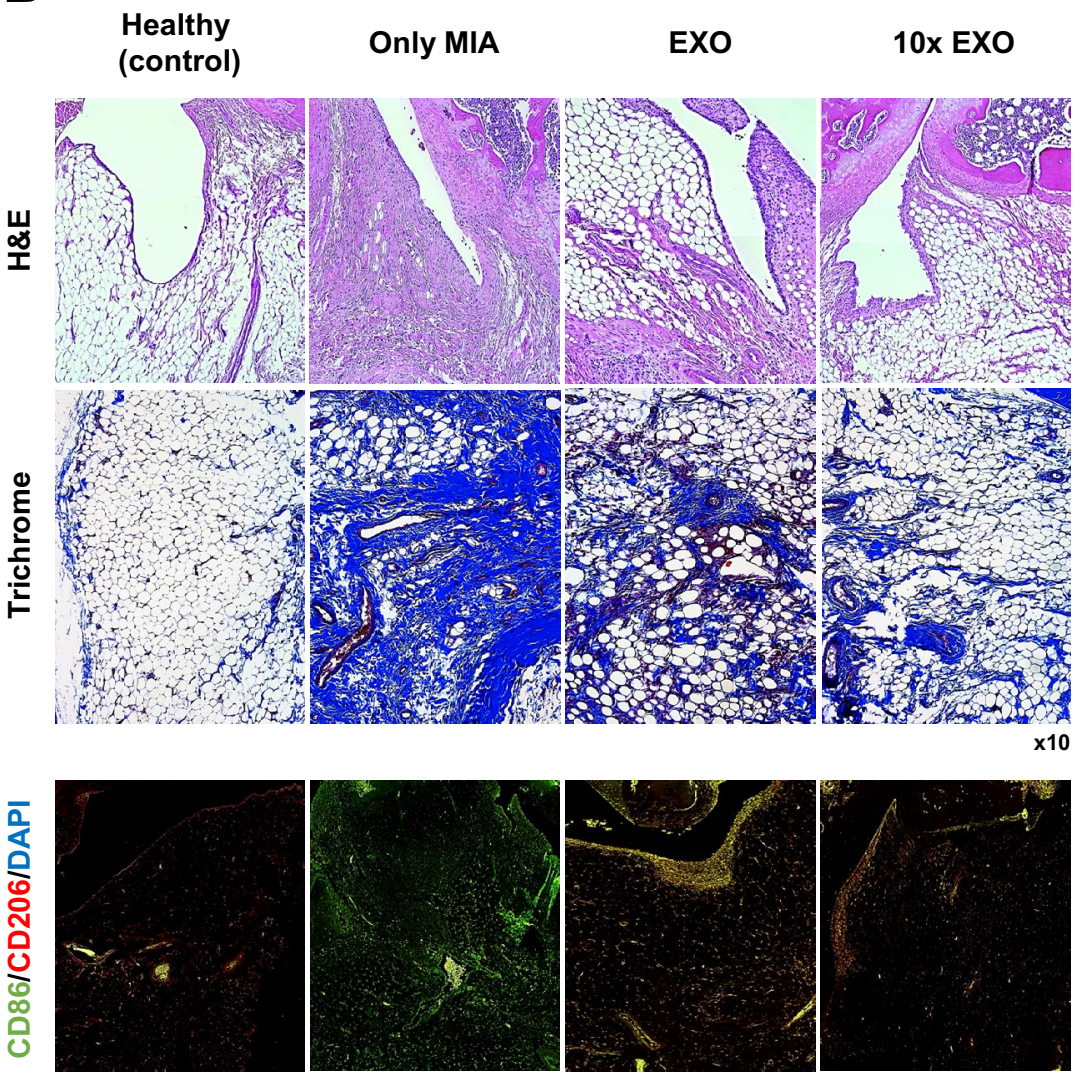

$\times 20$
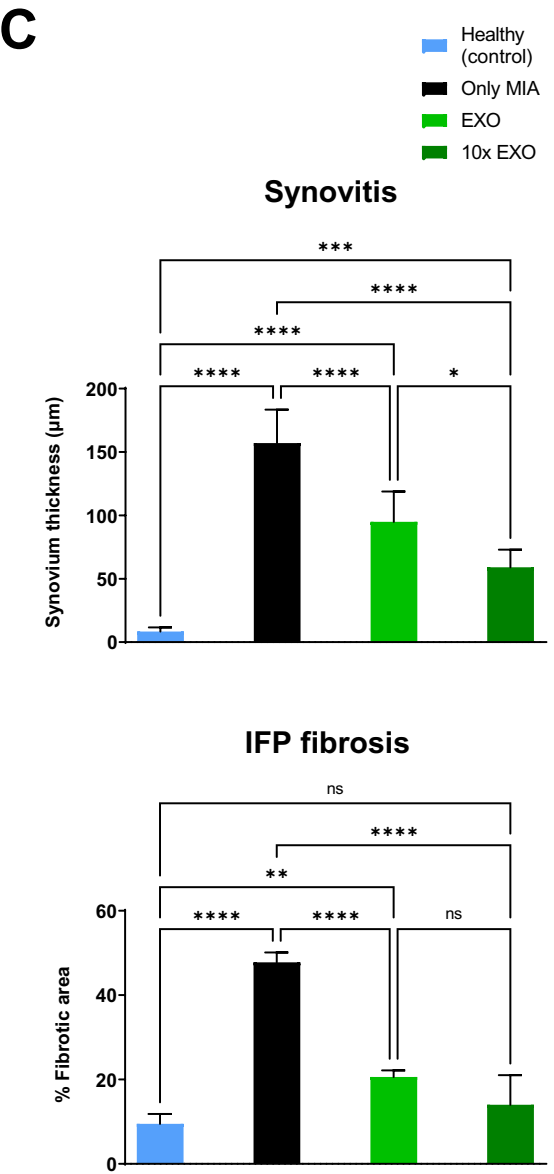

Macrophage polarization

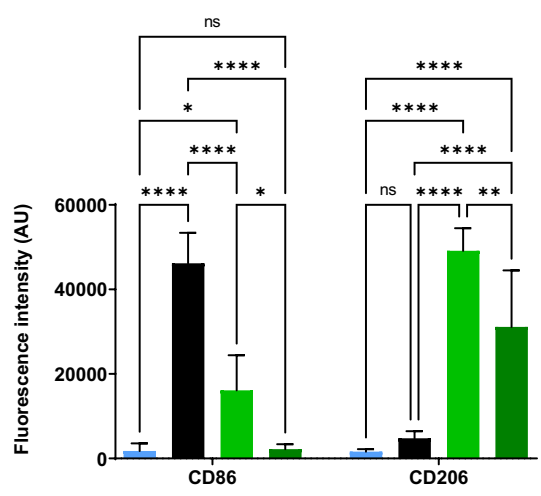

Figure 6. IFP-MSC EXOs effectively reverse synovitis/IFP fibrosis and polarize macrophages towards an alternatively activated-M2 phenotype in vivo. (A) Schematic indicating the generation of acute synovitis/IFP fibrosis rat model, IFP-MSC EXOs therapeutic intervention and chronological evaluation. (B,C) Hematoxylin/ Eosin staining (top panel), Masson's trichrome staining (middle panel) and CD86/CD206 immunolocalization (lower panel) in sagitally-sectioned knees of representative rats for healthy (control), diseased (only MIA) or IFP-MSC treated (with EXO and $10 \times \mathrm{EXO}$ ). Compared with only MIA injected group, which showed significant synovitis/IFP fibrosis and largely M1 $\left(\mathrm{CD}^{+} 6^{+}\right)$macrophages polarization, a striking correlation was found between IFP-MSC EXOs treatment and synovitis/fibrosis attenuation, and macrophages polarization towards alternatively activated-M2 $\left(\mathrm{CD} 206^{+}\right)$phenotype (ns: non-significant, ${ }^{*} p<0.05,{ }^{* *} p<0.01,{ }^{* * *} p<0.001$, $\left.{ }^{* * * *} p<0.0001\right)$.

Immunophenotype. Flow cytometric analysis was performed on P3 IFP-MSC $(\mathrm{n}=3)$. Briefly, $2.0 \times 10^{5}$ cells were labelled with monoclonal antibodies specific for: CD44, CD73, CD90, CD105, CD107a (Biolegend, 
San Diego, CA), CD146 (Miltenyi Biotec, Auburn, CA), CD166, NG2, HLA-DR (BD Biosciences, San Jose, CA), and the corresponding isotype controls. All samples included a Ghost Red Viability Dye (Tonbo Biosciences, San Diego, CA, USA). Data (20.000 events collected) were acquired using a Cytoflex S (Beckman Coulter, Brea, CA, USA) and analyzed using Kaluza analysis software (Beckman Coulter).

Isolation and validation of IFP-MSC derived exosomes. IFP-MSC derived exosomes (IFP-MSC EXOs) were isolated from IFP-MSC conditioned medium by a step-wise ultracentrifugation method and CD63immunomagnetic purification. Briefly, conditioned media from IFP-MSC groups cultured in exosome-depleted Ch-R medium ${ }^{58}$ are filtered through a $0.22 \mu \mathrm{m}$ filter to remove debris and large vesicles, and differentially centrifuged for $2000 \times g$ for $10 \mathrm{~min}, 10,000 \times g$ for $30 \mathrm{~min}$, and ultracentrifuged for $120,000 \times g$ for $16 \mathrm{hr}^{59}$. Pre-enriched exosome preparations were incubated with the Dynabeads ${ }^{\oplus}$-based Exosome-Human CD63 Isolation/Detection Reagent (Invitrogen) and using a magnetic separator, exosome preparations are further purified. Samples from each group were assessed for biophysical and biochemical characterization ${ }^{60}$.

Quantity and size determination were performed by nanoparticle tracking analysis (NTA) (NanoSight NS300, Malvern). Samples were diluted in PBS from 1:10 to 1:100 depending on the initial sample concentration. The software settings for analysis were: detection threshold 5; room temperature; number of frames 30 and measurement time $30 \mathrm{~s}$. The size distribution and particle concentration each represent the mean of five individual measurements.

Exosomes presence was validated by CD9 (Invitrogen) expression in $\mathrm{CD}^{+} 3^{+}$-gated particles by flow cytometry. Data (20.000 events collected) were acquired using a Cytoflex S (Beckman Coulter) and analyzed using Kaluza analysis software (Beckman Coulter).

The functional assessment of IFP-MSC EXOs was performed in two concentrations corresponding to exosomes secreted from $5 \times 10^{5}(\mathrm{EXO})$ and $5 \times 10^{6}(10 \times \mathrm{EXO})$ IFP-MSC. For IFP-MSC EXOs tracking, exosomes were stained with PKH26 red fluorescent membrane staining kit (Fluorescent Cell Linker Kits, Sigma) according to manufacturer's instructions and co-cultured with target cells in functional assessments.

miRNA profile of IFP-MSC EXOs. miRNA and protein were extracted from IFP-EXOs using Total Exosome RNA and Protein Isolation Kit (Thermo Fisher Scientific) according to manufacturer's instructions. Total exosome miRNA $(1 \mu \mathrm{g})$ was used for first-strand cDNA synthesis with All-in-One miRNA First-Strand cDNA Synthesis Kit (GeneCopoeia, Rockville, MD).

Pre-designed human MSC exosome 166 miRNA qPCR arrays (GeneCopoeia) were performed using $1000 \mathrm{ng}$ cDNA per IFP-MSC sample $(\mathrm{n}=2)$, and processed using StepOne Real-time thermocycler (Applied Biosystems, LLC). Data analysis was performed using qPCR result with GeneCopoeia’s online Data Analysis System (http:// www.genecopoeia.com/product/qpcr/analyse/). Mean values were normalized to small nucleolar RNA, C/D box 48 (SNORD48), expression levels were calculated using the $2^{-\Delta \mathrm{Ct}}$ method. Putative miRNA interactomes were generated using a miRNet centric network visual analytics platform (https://www.mirnet.ca/). The miRNA target gene data were collected from well-annotated database miRTarBase v8.0 and miRNA-gene interactome network refining was performed with 2.0 betweenness cut-off. Values (with 34 cycles cut-off point) were represented in a topology miRNA-gene interactome network using force atlas layout and hypergeometric test algorithm. InfoMap algorithm was also used to identify miRNAs with higher node degree that act as hubs in a gene network.

Protein profile of IFP-MSC EXOs. Multiplex protein arrays of 35 cytokines and 41 growth factors (Ray$\mathrm{Bio}^{\oplus} \mathrm{C}$-Series, RayBiotech, Peachtree Corners, GA) were used to determine IFP-MSC EXOs protein cargo $(\mathrm{n}=2)$. One $\mathrm{mL}$ of IFP-MSC EXOs protein extract was used for each assay following the manufacturer's instructions. Data shown represent 40 s exposure in FluorChem E chemiluminescence imaging system (ProteinSimple, San Jose, CA). Results were generated by quantifying the mean spot pixel density of each array using protein array analyser plugin using ImageJ software (Fiji/ImageJ, NIH website). The signal intensities were normalized with the background whereas separate signal intensity results represent the average pixel density of two spots per protein. Signal intensity for each protein spot is proportional to the relative concentration of the antigen in the sample.

Pathway analysis. Putative interactomes were generated by Search Tool for Retrieval of Interacting Genes/ Proteins (STRING 11.0; available from: http://string-db.org) database using interaction data from experiments, databases, neighbourhood in genome, gene fusions, co-occurrence across genomes, co-expression and textmining. An interaction confidence score of 0.4 was imposed to ensure high interaction probability. K-means clustering algorithm was used to organize proteins into 3 separate clusters per condition tested, discriminated by colours. Venn diagrams were used to demonstrate all possible relations between naïve and/or TIC-primed IFP-MSC cultured under all three different conditions for the significantly $(p<0.05)$ altered proteins. Functional enrichments related to biological process, Kyoto Encyclopedia of Genes and Genomes (KEGG) pathways, and reactome pathways were presented in radar graphs for proteins tested.

Synoviocyte inflammation assay. Passage 1 synoviocytes (SYN) expanded in synoviocyte medium (ScienCell, Carlsbad, CA) were subsequently primed with TIC inflammatory/fibrotic cocktail (15 ng/ml TNFa, $10 \mathrm{ng} / \mathrm{ml} \mathrm{IFN} \gamma, 10 \mathrm{ng} / \mathrm{ml} \mathrm{CTGF)} \mathrm{for} 72 \mathrm{~h}$. On day 3, synoviocyte/exosome (SYN/ IFP-MSC EXOs) co-cultures were performed using EXO and $10 \times \mathrm{EXO}$ for each sample. Co-cultures were fed with synoviocyte medium or synoviocyte medium + TIC inflammatory/fibrotic cocktail for $72 \mathrm{~h}$ according to the initial non-induced or TICprimed cohorts designation. 
CCK-8 cytotoxicity assay (Cell Counting Kit-8, Sigma) was performed in non-induced or TIC-primed SYN and SYN/ IFP-MSC EXOs according to manufacturer's instructions. EXO and $10 \times$ EXO cytotoxicity was determined by measuring optical densities of individual wells at $450 \mathrm{~nm}$ (SpectraMax M5 spectrophotometer, Molecular Devices, San Jose, CA).

RNA extraction from SYN cultures was performed using the RNeasy Mini Kit (Qiagen, Frederick, MD) according to manufacturer's instructions. Total RNA $(1 \mu \mathrm{g})$ was used for reverse transcription with SuperScript ${ }^{\mathrm{Tix}}$ $\mathrm{VILO}^{\mathrm{m}}$ cDNA synthesis kit (Invitrogen). A pre-designed 28 gene Taqman low density cytokine array (TLDA, Applied Biosystems) was performed using $1000 \mathrm{ng}$ cDNA per culture and processed using StepOne Real-time thermocycler (Applied Biosystems, LLC). Data analysis was performed using DataAssist software v2.0 (Applied Biosystems, LLC). Mean values were normalized to GAPDH, expression levels were calculated using the $2^{-\Delta \mathrm{Ct}}$ method. Values were represented in a fold change heatmap as the relative fold change of the TIC SYN/EXO or TIC SYN $/ 10 \times$ EXO to TIC SYN (reference sample, $2^{-\Delta \mathrm{Ct}}=\mathrm{X}$ sample/X reference sample).

Secretory profiles of non-induced and TIC-primed cohorts were evaluated using multiplex protein arrays of 40 cytokines (RayBio ${ }^{\circ} \mathrm{C}$-Series, RayBiotech). One $\mathrm{mL}$ of conditioned media was used for each assay following the manufacturer's instructions. Data shown represent 40 s exposure in FluorChem E chemiluminescence imaging system (ProteinSimple, San Jose, CA). Results were generated by quantifying the mean spot pixel density of each array using protein array analyser plugin using ImageJ software (Fiji/ImageJ, NIH website). Signal intensities were normalized with the background whereas separate signal intensity results represent the average pixel density of two spots per protein. The signal intensity for each protein spot is proportional to the relative concentration of the antigen in the sample.

Macrophage polarization assay. Human monocytes (THP-1, ATCC) were differentiated into macrophages using PMA/IO (Phorbol 12-myristate 13-acetate/Ionomycin) and polarized to M1 macrophages by M1-macrophage generation medium (PromoCell). PMA/IO-stimulated THP-1/IFP-MSC EXOs co-cultures were performed for 2 days using EXO and $10 \times \mathrm{EXO}$. Generated macrophages inflammation-related protein profiling was performed by multiplex protein arrays whereas their polarization status was assessed using macrophage polarization qPCR array (ScienCell).

A CCK-8 cytotoxicity assay (Cell Counting Kit-8, Sigma) was performed in non-induced or PMA/IO-primed THP-1 and THP-1/IFP-MSC EXOs according to manufacturer's instructions. EXO and $10 \times$ EXO cytotoxicity was determined by measuring optical densities of individual wells at $450 \mathrm{~nm}$ (SpectraMax M5 spectrophotometer, Molecular Devices, San Jose, CA).

Secretory profiles of PMA/IO-primed cohorts were evaluated using multiplex protein arrays of 40 cytokines (RayBio ${ }^{\circ} \mathrm{C}$-Series, RayBiotech). One $\mathrm{mL}$ of conditioned media was used for each assay following the manufacturer's instructions. Data shown represent $40 \mathrm{~s}$ exposure in FluorChem E chemiluminescence imaging system (ProteinSimple, San Jose, CA). Results were generated by quantifying the mean spot pixel density of each array using protein array analyser plugin using ImageJ software (Fiji/ImageJ, NIH website). The signal intensities were normalized with the background whereas separate signal intensity results represent the average pixel density of two spots per protein. The signal intensity for each protein spot is proportional to the relative concentration of the antigen in the sample.

RNA extraction from THP-1 cultures was performed using the RNeasy Mini Kit (Qiagen, Frederick, MD) according to manufacturer's instructions. Total RNA $(1 \mu \mathrm{g})$ was used for reverse transcription with SuperScript ${ }^{\mathrm{mix}}$ VILO $^{m} \mathrm{cDNA}$ synthesis kit (Invitrogen). A pre-designed 40 gene Human Macrophage Polarization array (GeneQuery $^{\mathrm{mm}}$ Human Macrophage Polarization Markers qPCR Array Kit, ScienCell) was performed using 1000 ng cDNA per culture and processed using StepOne Real-time thermocycler (Applied Biosystems, LLC). Mean values were normalized to GAPDH, expression levels were calculated using the $2^{-\Delta \mathrm{Ct}}$ method. Values were represented in a stacked bar plot for M0, M1, and M2 polarization as the relative fold change of the PMA/IO + THP-1/EXO or PMA/IO + THP-1/10xEXO to PMA/IO + THP-1 (reference sample, $2-\Delta \mathrm{Ct}=\mathrm{X}$ sample/X reference sample).

Mono-iodoacetate model of acute synovial/IFP inflammation. All animal experiments were performed in accordance with relevant guidelines and regulations. The animal protocol was approved by the Institutional Animal Care and Use Committee (IACUC) of the University of Miami, USA (approval no. 21-030 LF) and conducted in accordance to the ARRIVE guidelines ${ }^{61}$. Twelve (\#12) 10-week old male Sprague Dawley rats (mean weight $250 \mathrm{~g}$ ) were used. The animals were housed to acclimate for 1 week before the experiment initiation. One rat was housed per cage in a sanitary, ventilated room with controlled temperature, humidity, and under a 12/12 h light/dark cycle with food and water provided ad libitum.

Acute synovial/IFP inflammation was generated by intra-articular injection of $1 \mathrm{mg}$ of mono-iodoacetate (MIA) in $50 \mu \mathrm{l}$ of saline into the right knee. Briefly, under isoflurane inhalation anesthesia, rat knees were flexed $90^{\circ}$ and MIA was injected into the medial side of the joint with a $27 \mathrm{G}$ needle using the patellar ligament and articular line as anatomical references. Four (4) days later, a single intra-articular injection of EXO or $10 \times \mathrm{EXO}$ in $50 \mu \mathrm{l}$ of Euro-Collins solution (MediaTech) was performed (similar injection technique), having as control: (1) rat knees receiving MIA but not IFP-EXOs (Only MIA group); and (2) healthy rat knees. Animals were sacrificed at day 4 after IFP-EXOs injection ( $\mathrm{d} 8$ in total). This short exposure to MIA has been shown to induce inflammatory changes within the synovium and adjacent IFP ${ }^{53}$.

Cytochemical staining and CD86/CD206 immunolocalization in situ. Rat knee joints were harvested by cutting the femur and tibia/fibula $1 \mathrm{~cm}$ above and below the joint line, muscles were dissected and removed, and joints were fixed with $10 \%$ neutral buffered formalin (Sigma-Aldrich) for 14 days at room temperature. Knee joints were decalcified, cut along the sagittal plane in half, embedded in paraffin, and serial $4 \mu \mathrm{m}$ 
sections obtained. Hematoxylin and Eosin $(\mathrm{H} \& \mathrm{E})$ staining was performed to evaluate the gross structure and morphology of the knee joints. Masson's trichrome staining for collagen-rich fibrotic areas was used to evaluate for extent of fibrosis within the fat pad tissue. Microscope images of cytochemically stained tissues were acquired using $\times 10$ objective Leica DMi8 microscope with Leica X software (Leica). Based on histochemical stainings, tissue synovitis/fibrosis was evaluated in 3 rat knees per condition and 4 microscopy fields per knee with Fiji/ ImageJ software.

For anti-CD86 and CD206 immunofluorescence staining, sections were incubated with $1 \times$ citrate buffer solution at $60{ }^{\circ} \mathrm{C}$ overnight for antigen retrieval, permeabilised with $1 \times \mathrm{PBS}+0.2 \%$ Triton X-100 for 20 min at room temperature, and incubated with blocking buffer $(1 \times \mathrm{PBS}+0.1 \%$ Triton $\mathrm{X}-100$ with $10 \%$ rabbit serum $)$ for $1 \mathrm{~h}$ at room temperature. In between different treatments sections were washed with $1 \times \mathrm{PBS}$. Mouse anti-rat CD86 monoclonal antibody and rabbit anti-rat CD206 polyclonal antibody (both from Abcam) were prepared in blocking buffer (1:500) and sections were incubated at $4{ }^{\circ} \mathrm{C}$ overnight. Sections were washed with $1 \times \mathrm{PBS}+0.01 \%$ Triton X-100 and incubated for $2 \mathrm{~h}$ with secondary antibodies containing Alexa Fluor488 conjugated goat anti-mouse and Alexa Fluor647 conjugated goat anti-rabbit IgG antibodies (Thermo Fisher Scientific) at room temperature. Controls were incubated with secondary antibodies only. All sections were rinsed with $1 \times$ PBS, mounted in prolong gold antifade reagent with DAPI (Invitrogen), and microscope images were acquired using Leica DMi8 microscope with Leica X software (Leica).

Statistical analysis. Normal distribution of values was assessed by the Kolmogorov-Smirnov normality test. Statistical analysis was performed using paired and unpaired Student's $t$-test for normally distributed data and Wilcoxon (for paired data) or Mann Whitney (for unpaired data) test. In the presence of a non-normal distribution of the data, a one-way ANOVA was used for multiple comparisons. All tests were performed with GraphPad Prism v7.03 (GraphPad Software, San Diego, CA). Level of significance was set at $p<0.05$.

Received: 3 December 2021; Accepted: 21 February 2022

Published online: 04 March 2022

\section{References}

1. Macchi, V. et al. The infrapatellar fat pad and the synovial membrane: An anatomo-functional unit. J. Anat. 233, 146-154. https:// doi.org/10.1111/joa.12820 (2018).

2. Sokolove, J. \& Lepus, C. M. Role of inflammation in the pathogenesis of osteoarthritis: Latest findings and interpretations. Ther. Adv. Musculoskelet. Dis. 5, 77-94. https://doi.org/10.1177/1759720X12467868 (2013).

3. Lent, P. et al. Active involvement of alarmins S100A8 and S100A9 in the regulation of synovial activation and joint destruction during mouse and human osteoarthritis. Arthritis Rheum. 64, 1466-1476. https://doi.org/10.1002/art.34315 (2011).

4. Schelbergen, R. F. et al. Alarmins S100A8 and S100A9 elicit a catabolic effect in human osteoarthritic chondrocytes that is dependent on Toll-like receptor 4. Arthritis Rheum. 64, 1477-1487. https://doi.org/10.1002/art.33495 (2011).

5. Sohn, D. H. et al. Plasma proteins present in osteoarthritic synovial fluid can stimulate cytokine production via Toll-like receptor 4. Arthritis Res. Ther. 14, R7. https://doi.org/10.1186/ar3555 (2012).

6. Eymard, F. et al. Induction of an inflammatory and prodegradative phenotype in autologous fibroblast-like synoviocytes by the infrapatellar fat pad from patients with knee osteoarthritis. Arthritis Rheumatol. 66, 2165-2174. https://doi.org/10.1002/art.38657 (2014).

7. Caplan, A. I. \& Correa, D. The MSC: An injury drugstore. Cell Stem Cell 9, 11-15 (2011).

8. Tangchitphisut, P. et al. Infrapatellar fat pad: An alternative source of adipose-derived mesenchymal stem cells. Arthritis 2016, 4019873. https://doi.org/10.1155/2016/4019873 (2016).

9. Galipeau, J. et al. International Society for Cellular Therapy perspective on immune functional assays for mesenchymal stromal cells as potency release criterion for advanced phase clinical trials. Cytotherapy 18, 151-159. https://doi.org/10.1016/j.jcyt.2015. 11.008 (2016).

10. Kotani, T. et al. Anti-inflammatory and anti-fibrotic effects of intravenous adipose-derived stem cell transplantation in a mouse model of bleomycin-induced interstitial pneumonia. Sci. Rep. 8, 454. https://doi.org/10.1038/s41598-017-17991-x (2018).

11. Uccelli, A. \& Rosbo, N. K. The immunomodulatory function of mesenchymal stem cells: Mode of action and pathways. Ann. N. Y. Acad. Sci. 1351, 114-126. https://doi.org/10.1111/nyas.12815 (2015).

12. Stagg, J. \& Galipeau, J. Mechanisms of immune modulation by mesenchymal stromal cells and clinical translation. Curr. Mol. Med. 13, 856-867. https://doi.org/10.2174/1566524011313050016 (2013).

13. Kouroupis, D. et al. Infrapatellar fat pad-derived MSC response to inflammation and fibrosis induces an immunomodulatory phenotype involving CD10-mediated Substance P degradation. Sci. Rep. 9, 10864. https://doi.org/10.1038/s41598-019-47391-2 (2019).

14. Kouroupis, D., Best, T. M., Kaplan, L. D., Correa, D. \& Griswold, A. J. Single-cell RNA-sequencing identifies infrapatellar fat pad macrophage polarization in acute synovitis/fat pad fibrosis and cell therapy. Bioengineering 8, 166 (2021).

15. Sturiale, S. et al. Neutral endopeptidase (EC 3.4.24.11) terminates colitis by degrading substance P. Proc. Natl. Acad. Sci. USA 96, 11653-11658. https://doi.org/10.1073/pnas.96.20.11653 (1999).

16. Nikfarjam, S., Rezaie, J., Zolbanin, N. M. \& Jafari, R. Mesenchymal stem cell derived-exosomes: A modern approach in translational medicine. J. Transl. Med. 18, 449. https://doi.org/10.1186/s12967-020-02622-3 (2020).

17. Witwer, K. W. et al. Defining mesenchymal stromal cell (MSC)-derived small extracellular vesicles for therapeutic applications. J. Extracell. Vesicles 8, 1609206. https://doi.org/10.1080/20013078.2019.1609206 (2019).

18. van Balkom, B. W. M., Gremmels, H., Giebel, B. \& Lim, S. K. Proteomic signature of mesenchymal stromal cell-derived small extracellular vesicles. Proteomics 19, 1800163. https://doi.org/10.1002/pmic.201800163 (2019).

19. Kouroupis, D., Sanjurjo-Rodriguez, C., Jones, E. \& Correa, D. Mesenchymal stem cell functionalization for enhanced therapeutic applications. Tissue Eng. Part B. 25, 55-77. https://doi.org/10.1089/ten.teb.2018.0118 (2018).

20. Kouroupis, D. et al. Regulatory-compliant conditions during cell product manufacturing enhance in vitro immunomodulatory properties of infrapatellar fat pad-derived mesenchymal stem/stromal cells. Cytotherapy 22, 677-689. https://doi.org/10.1016/j. jcyt.2020.06.007 (2020). 
21. Kouroupis, D., Bowles, A. C., Best, T. M., Kaplan, L. D. \& Correa, D. CD10/neprilysin enrichment in infrapatellar fat pad-derived mesenchymal stem cells under regulatory-compliant conditions: Implications for efficient synovitis and fat pad fibrosis reversal. Am. J. Sports Med. 48, 2013-2027. https://doi.org/10.1177/0363546520917699 (2020).

22. Bourin, P. et al. Stromal cells from the adipose tissue-derived stromal vascular fraction and culture expanded adipose tissuederived stromal/stem cells: A joint statement of the International Federation for Adipose Therapeutics and Science (IFATS) and the International Society for Cellular Therapy (ISCT). Cytotherapy 15, 641-648. https://doi.org/10.1016/j.jcyt.2013.02.006 (2013).

23. Dominici, M. et al. Minimal criteria for defining multipotent mesenchymal stromal cells. The International Society for Cellular Therapy position statement. Cytotherapy 8, 315-317 (2006).

24. Hindle, P., Khan, N., Biant, L. \& Péault, B. The infrapatellar fat pad as a source of perivascular stem cells with increased chondrogenic potential for regenerative medicine. Stem Cells Transl. Med. 6, 77-87. https://doi.org/10.5966/sctm.2016-0040 (2017).

25. Tormin, A. et al. CD146 expression on primary nonhematopoietic bone marrow stem cells is correlated with in situ localization. Blood 117, 5067-5077. https://doi.org/10.1182/blood-2010-08-304287 (2011).

26. Crisan, M. et al. A perivascular origin for mesenchymal stem cells in multiple human organs. Cell Stem Cell 3, 301-313. https:// doi.org/10.1016/j.stem.2008.07.003 (2008).

27. Théry, C. et al. Minimal information for studies of extracellular vesicles 2018 (MISEV2018): A position statement of the International Society for Extracellular Vesicles and update of the MISEV2014 guidelines. J. Extracell. Vesicles 7, 1535750. https://doi.org/ 10.1080/20013078.2018.1535750 (2018).

28. Bartel, D. P. MicroRNAs: Genomics, biogenesis, mechanism, and function. Cell 116, 281-297. https://doi.org/10.1016/S00928674(04)00045-5 (2004).

29. Eulalio, A., Huntzinger, E. \& Izaurralde, E. Getting to the root of miRNA-mediated gene silencing. Cell 132, 9-14. https://doi.org/ 10.1016/j.cell.2007.12.024 (2008)

30. Tahamtan, A., Teymoori-Rad, M., Nakstad, B. \& Salimi, V. Anti-inflammatory micrornas and their potential for inflammatory diseases treatment. Front. Immunol. https://doi.org/10.3389/fimmu.2018.01377 (2018).

31. Ni, Z. et al. Exosomes: Roles and therapeutic potential in osteoarthritis. Bone Res. 8, 25. https://doi.org/10.1038/s41413-020-0100-9 (2020).

32. Wu, J. et al. miR-100-5p-abundant exosomes derived from infrapatellar fat pad MSCs protect articular cartilage and ameliorate gait abnormalities via inhibition of mTOR in osteoarthritis. Biomaterials 206, 87-100. https://doi.org/10.1016/j.biomaterials.2019. 03.022 (2019).

33. Naqvi, A. R., Fordham, J. B. \& Nares, S. miR-24, miR-30b, and miR-142-3p regulate phagocytosis in myeloid inflammatory cells. J. Immunol. 194, 1916-1927. https://doi.org/10.4049/jimmunol.1401893 (2015).

34. Zhang, Y. et al. MicroRNA-142-3p relieves neuropathic pain by targeting high mobility group box 1. Int. J. Mol. Med. 41, 501-510. https://doi.org/10.3892/ijmm.2017.3222 (2018).

35. Curtale, G. et al. An emerging player in the adaptive immune response: MicroRNA-146a is a modulator of IL-2 expression and activation-induced cell death in T lymphocytes. Blood 115, 265-273. https://doi.org/10.1182/blood-2009-06-225987 (2010).

36. Jurkin, J. et al. miR-146a is differentially expressed by myeloid dendritic cell subsets and desensitizes cells to TLR2-dependent activation. J. Immunol. 184, 4955-4965. https://doi.org/10.4049/jimmunol.0903021 (2010).

37. Foley, N. H. \& O'Neill, L. A. miR-107: A Toll-like receptor-regulated miRNA dysregulated in obesity and type II diabetes. J. Leukoc. Biol. 92, 521-527. https://doi.org/10.1189/jlb.0312160 (2012).

38. Peng, Y., Zhao, J.-L., Peng, Z.-Y., Xu, W.-F. \& Yu, G.-L. Exosomal miR-25-3p from mesenchymal stem cells alleviates myocardial infarction by targeting pro-apoptotic proteins and EZH2. Cell Death Dis. 11, 317. https://doi.org/10.1038/s41419-020-2545-6 (2020).

39. Zhu, C., Chen, T. \& Liu, B. Inhibitory effects of miR-25 targeting HMGB1 on macrophage secretion of inflammatory cytokines in sepsis. Oncol. Lett. 16, 5027-5033. https://doi.org/10.3892/ol.2018.9308 (2018).

40. Barajas-Gómez, B. A. et al. Relationship of inflammatory profile of elderly patients serum and senescence-associated secretory phenotype with human breast cancer cells proliferation: Role of IL6/IL8 ratio. Cytokine 91, 13-29. https://doi.org/10.1016/j.cyto. 2016.12.001 (2017).

41. Chinnadurai, R. et al. Potency analysis of mesenchymal stromal cells using a combinatorial assay matrix approach. Cell Rep. 22, 2504-2517. https://doi.org/10.1016/j.celrep.2018.02.013 (2018).

42. Knight, B. E. et al. TIMP-1 attenuates the development of inflammatory pain through MMP-dependent and receptor-mediated cell signaling mechanisms. Front. Mol. Neurosci. https://doi.org/10.3389/fnmol.2019.00220 (2019).

43. Lee, E.-J. \& Kim, H.-S. The anti-inflammatory role of tissue inhibitor of metalloproteinase-2 in lipopolysaccharide-stimulated microglia. J. Neuroinflamm. 11, 116-116. https://doi.org/10.1186/1742-2094-11-116 (2014).

44. Kubiczkova, L., Sedlarikova, L., Hajek, R. \& Sevcikova, S. TGF- $\beta$ : An excellent servant but a bad master. J. Transl. Med. 10, 183. https://doi.org/10.1186/1479-5876-10-183 (2012).

45. Ge, Q. et al. VEGF secreted by mesenchymal stem cells mediates the differentiation of endothelial progenitor cells into endothelial cells via paracrine mechanisms. Mol. Med. Rep. 17, 1667-1675. https://doi.org/10.3892/mmr.2017.8059 (2018).

46. Prieto-Potin, I., Largo, R., Roman-Blas, J. A., Herrero-Beaumont, G. \& Walsh, D. A. Characterization of multinucleated giant cells in synovium and subchondral bone in knee osteoarthritis and rheumatoid arthritis. BMC Musculoskelet. Disord. 16, 226. https:// doi.org/10.1186/s12891-015-0664-5 (2015).

47. Tu, J. et al. Ontology and function of fibroblast-like and macrophage-like synoviocytes: How do they talk to each other and can they be targeted for rheumatoid arthritis therapy?. Front. Immunolo. https://doi.org/10.3389/fimmu.2018.01467 (2018).

48. Greif, D. N. et al. Infrapatellar fat pad/synovium complex in early-stage knee osteoarthritis: Potential new target and source of therapeutic mesenchymal stem/stromal cells. Front. Bioeng. Biotechnol. https://doi.org/10.3389/fbioe.2020.00860 (2020).

49. Meng, Q. \& Qiu, B. Exosomal microRNA-320a derived from mesenchymal stem cells regulates rheumatoid arthritis fibroblast-like synoviocyte activation by suppressing CXCL9 expression. Front. Physiol. 11, 441-441. https://doi.org/10.3389/fphys.2020.00441 (2020).

50. Orecchioni, M., Ghosheh, Y., Pramod, A. B. \& Ley, K. Macrophage polarization: Different gene signatures in M1(LPS+) vs Classically and M2(LPS-) vs. alternatively activated macrophages. Front. Immunol. 10, 1084. https://doi.org/10.3389/fimmu.2019.01084 (2019).

51. Sierra-Filardi, E. et al. CCL2 shapes macrophage polarization by GM-CSF and M-CSF: Identification of CCL2/CCR2-dependent gene expression profile. J. Immunol. 192, 3858-3867. https://doi.org/10.4049/jimmunol.1302821 (2014).

52. Bowles, A. C. et al. Signature quality attributes of CD146+ mesenchymal stem/stromal cells correlate with high therapeutic and secretory potency. Stem Cells 38, 1034-1049. https://doi.org/10.1002/stem.3196 (2020).

53. Udo, M. et al. Monoiodoacetic acid induces arthritis and synovitis in rats in a dose- and time-dependent manner: Proposed model-specific scoring systems. Osteoarthritis Cartilage 24, 1284-1291. https://doi.org/10.1016/j.joca.2016.02.005 (2016).

54. Maglaviceanu, A., Wu, B. \& Kapoor, M. Fibroblast-like synoviocytes: Role in synovial fibrosis associated with osteoarthritis. Wound Repair Regen. 29, 642-649. https://doi.org/10.1111/wrr.12939 (2021).

55. Bondeson, J. et al. The role of synovial macrophages and macrophage-produced mediators in driving inflammatory and destructive responses in osteoarthritis. Arthritis Rheum 62, 647-657. https://doi.org/10.1002/art.27290 (2010).

56. Bernardo, M. E. \& Fibbe, W. E. Mesenchymal stromal cells: Sensors and switchers of inflammation. Cell Stem Cell 13, 392-402. https://doi.org/10.1016/j.stem.2013.09.006 (2013). 
57. Woo, C. H. et al. Small extracellular vesicles from human adipose-derived stem cells attenuate cartilage degeneration. J. Extracell. Vesicles 9, 1735249. https://doi.org/10.1080/20013078.2020.1735249 (2020).

58. Pachler, K. et al. A Good Manufacturing Practice-grade standard protocol for exclusively human mesenchymal stromal cell-derived extracellular vesicles. Cytotherapy 19, 458-472. https://doi.org/10.1016/j.jcyt.2017.01.001 (2017).

59. Webber, J. \& Clayton, A. How pure are your vesicles?. J. Extracell. Vesicles https://doi.org/10.3402/jev.v2i0.19861 (2013).

60. Rohde, E., Pachler, K. \& Gimona, M. Manufacturing and characterization of extracellular vesicles from umbilical cord-derived mesenchymal stromal cells for clinical testing. Cytotherapy 21, 581-592. https://doi.org/10.1016/j.jcyt.2018.12.006 (2019).

61. Kilkenny, C., Browne, W. J., Cuthill, I. C., Emerson, M. \& Altman, D. G. Improving bioscience research reporting: The ARRIVE guidelines for reporting animal research. PLoS Biol. 8, e1000412. https://doi.org/10.1371/journal.pbio.1000412 (2010).

\section{Acknowledgements}

The authors are in gratitude with the Soffer Family Foundation and the DRI Foundation for their generous funding support. These funding sources were not involved in any step of the study design, collection, analysis, or interpretation of the data, or writing of the manuscript.

\section{Author contributions}

D.K. and T.M.B. Conceptualization; D.K. methodology, software, validation, formal analysis, investigation, data curation; D.K. writing-original draft preparation; D.K. and T.M.B. writing-review and editing; L.D.K. and T.M.B. funding acquisition. All authors have read and agreed to the published version of the manuscript.

\section{Competing interests}

The authors declare no competing interests.

\section{Additional information}

Supplementary Information The online version contains supplementary material available at https://doi.org/ 10.1038/s41598-022-07569-7.

Correspondence and requests for materials should be addressed to D.K.

Reprints and permissions information is available at www.nature.com/reprints.

Publisher's note Springer Nature remains neutral with regard to jurisdictional claims in published maps and institutional affiliations.

Open Access This article is licensed under a Creative Commons Attribution 4.0 International License, which permits use, sharing, adaptation, distribution and reproduction in any medium or format, as long as you give appropriate credit to the original author(s) and the source, provide a link to the Creative Commons licence, and indicate if changes were made. The images or other third party material in this article are included in the article's Creative Commons licence, unless indicated otherwise in a credit line to the material. If material is not included in the article's Creative Commons licence and your intended use is not permitted by statutory regulation or exceeds the permitted use, you will need to obtain permission directly from the copyright holder. To view a copy of this licence, visit http://creativecommons.org/licenses/by/4.0/.

(C) The Author(s) 2022 